This item was submitted to Loughborough's Research Repository by the author.

Items in Figshare are protected by copyright, with all rights reserved, unless otherwise indicated.

\title{
A multiscale finite element space-time discretization method for transient transport phenomena using bubble functions
}

PLEASE CITE THE PUBLISHED VERSION

PUBLISHER

(C) Elsevier

VERSION

AM (Accepted Manuscript)

LICENCE

CC BY-NC-ND 4.0

\section{REPOSITORY RECORD}

Nassehi, Vahid, and Mahmoud Parvazinia. 2009. "A Multiscale Finite Element Space-time Discretization Method for Transient Transport Phenomena Using Bubble Functions”. figshare.

https://hdl.handle.net/2134/4741. 
This item was submitted to Loughborough's Institutional Repository (https://dspace.lboro.ac.uk/) by the author and is made available under the following Creative Commons Licence conditions.

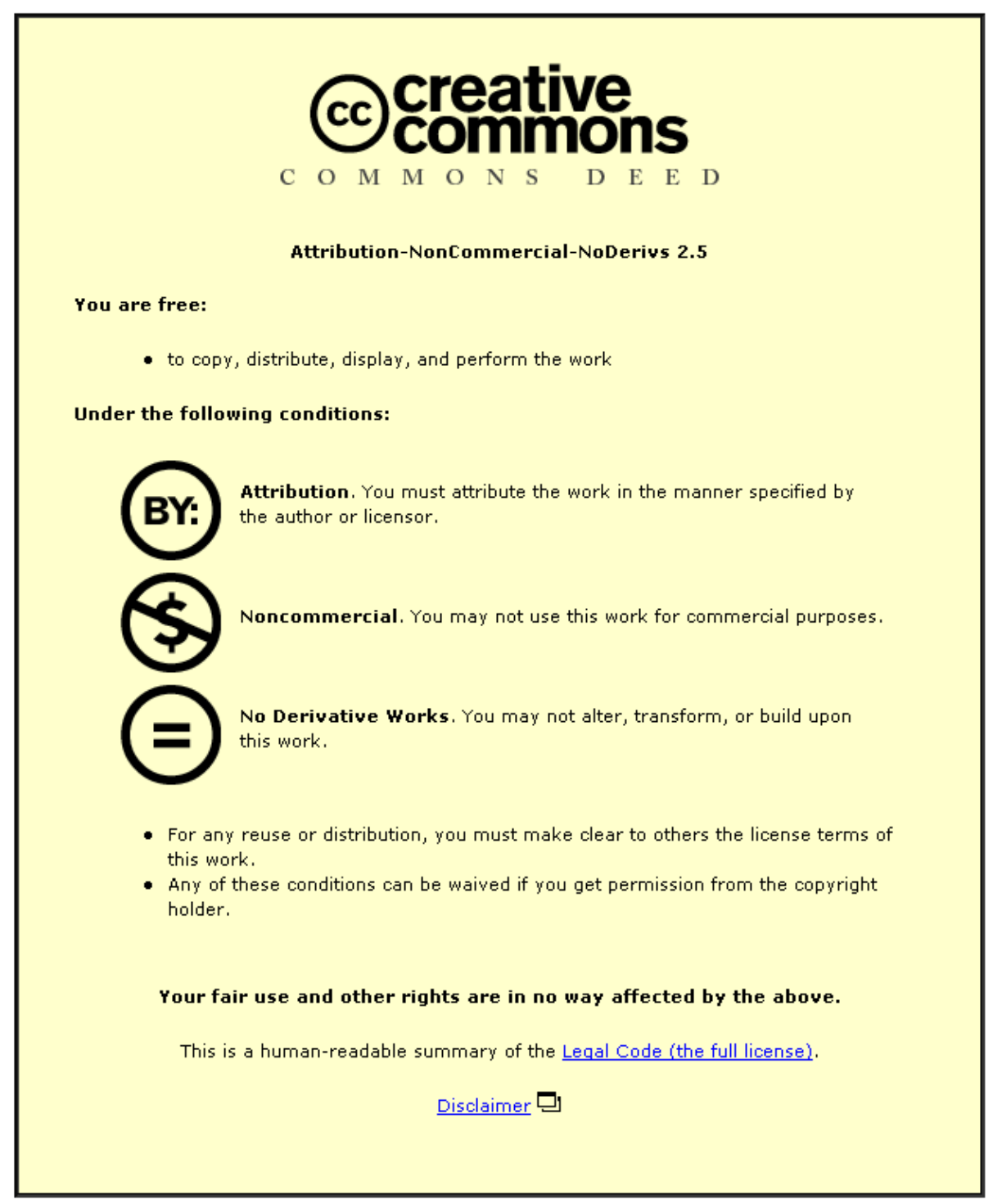

For the full text of this licence, please go to: http://creativecommons.org/licenses/by-nc-nd/2.5/ 


\title{
A multiscale finite element space-time discretization method for transient transport phenomena using bubble functions
}

\author{
V.Nassehi ${ }^{1}$, M.Parvazinia ${ }^{2}$ \\ 1- Department of Chemical Engineering, Loughborough University, Loughborough, \\ Leicestershire, LE11 3TU, UK \\ 2-Iran Polymer and Petrochemical Institute, P.O. Box: 14965/115, Tehran, Iran.
}

\begin{abstract}
A space-time finite element discretization method for unsteady transport phenomena is formulated using a variational multiscale finite element scheme. The described discretization is based on the utilization of bubble function enriched finite elements. The scheme is applied to model unsteady diffusion and convection-diffusion equations. It is shown that any temporal and spatial instabilities can be removed by appropriate enrichment of linear Lagrangian finite element approximations in the context of the standard Galerkin method. The proposed scheme is compared with widely used $\theta$ time stepping method. Numerical results generated by the proposed scheme are validated via their comparison with the analytical solution of a bench mark problem.
\end{abstract}

Keywords: Space-time discretization; Bubble functions; Multiscale finite element; unsteady problems, Transport phenomena.

\section{Introduction}

Stable and accurate numerical solution of transient transport problems has been the subject of numerous investigations. In particular, a large variety of time-stepping methods have been used to approximate transient models in conjunction with finite element method [1]. The basic issue in dealing with transient problems is to construct an optimum temporal discretizations in conjunction with a spatial discretization which is guaranteed to remain stable and accurate [2,3]. Generally finite element techniques for unsteady problems can be categorized as either decoupled formulations for space and time discretizations or coupled space-time formulations. In decoupled formulations, 
normally, a spatial finite element discretization is performed separately and temporal approximation is applied to the resulting equations. In contrast, in coupled procedures space-time discretization are carried out conjunctively [4]. Studies related to coupled space-time finite element discretizations can be trace for more than three decades. The works presented in [5-13] provide some of the notable example of such schemes.

Hughes and Stewart [14] proposed a space-time formulation for multiscale problems which is based on identical spatial and temporal discretizations. They extended the developed multiscale variational scheme originally proposed by Hughes [15] for steady problems, to time dependent situations. The main point of this scheme is that it yields a multiscale temporal approximation which can be used with larger time steps and hence is computationally cost effective.

In this paper, the scheme proposed by Hughes and Stewart [14] is extended to include bubble function enriched identical spatial and temporal Lagrangian approximations utilized in standard Galerkin finite element schemes. Multiscale variational approach is generally used to take into account the variations of field unknown ranging over different physical scales without using excessively refined computational girds [16]. Normally, in this approach the field unknown ( $T$ ) is divided into two parts as $T=T_{1}+T_{b}$, where $T_{b}$ is called fine, subgrid or unresolved scale while $T_{1}$ is called coarse or unresolved scale represented by standard polynomial finite element approximations. A possible way for generating subgrid scale model is based on the use of bubble functions. Bubble functions are, generally, high order polynomials which are zero on the element boundaries [17-24]. These functions can be used to enrich ordinary linear Lagrangian elements to generate higher order approximations without increasing the order of the elements in the nominal sense.

In the present study the global domain geometry is assumed to be constant and hence the finite element discretization is carried out over the entire space-time domain instead of each space-time intervals. Bench mark problems based on transport processes representing transient diffusion and transient convection-diffusion are solved and the numerical results are compared with their corresponding analytical solutions. The described comparison show that the proposed scheme is capable of yielding accurate and stable results. In addition, the results of the proposed method are compared with 
comparable values obtained by the widely used theta time stepping method to further validate the performance of the proposed scheme.

\section{Governing equations and boundary conditions}

Transient diffusion and convection-diffusion equations are considered. The transient convection-diffusion equation is written and for diffusion equation it is supposed that the convection coefficient is zero.

$\rho c\left(\frac{\partial T}{\partial t}+u . \nabla T\right)-k \nabla . \nabla T=f$

Where $T$ is independent variable $u$ is the velocity vector, $k$ is diffusivity, $\rho$ is density, $c$ is heat capacity and $f$ is a source term. $\nabla$ denotes the spatial gradient operator. Using the below mentioned dimensionless parameter:

$\left\{\begin{array}{l}T=T_{0}+T^{*}\left(T_{1}-T_{0}\right) \\ x^{*}=\frac{x}{h}, y^{*}=\frac{y}{h} \\ t^{*}=\frac{t}{t_{0}}\end{array}\right.$

where $T_{0}$ and $T_{1}$ are reference values for independent variable (e.g. temperature), $t_{0}$ is a characteristic time interval and $h$ is a characteristic length ( e.g width of the domain) dimensionless governing equation becomes:

$\frac{\partial T^{*}}{\partial t^{*}}+C \nabla T^{*}-D \nabla \cdot \nabla T^{*}=f^{*}$

in which $C$ and $D$ are dimensionless convection and diffusion coefficients respectively: 


$$
\left\{\begin{array}{l}
C=\frac{u t_{0}}{h} \\
D=\frac{k t_{0}}{h^{2} \rho c} \\
f^{*}=f \frac{t_{0}}{\rho c\left(T_{1}-T_{0}\right)}
\end{array}\right.
$$

Considering that in this work the same finite element discretization is used for both time and spatial dimensions, therefore we solve a two dimensional problem as follows:

$$
\frac{\partial T^{*}}{\partial t^{*}}+C \frac{\partial T^{*}}{\partial x^{*}}-D \frac{\partial^{2} T^{*}}{\partial x^{* 2}}=f^{*}
$$

Corresponding dimensionless boundary conditions for the rectangular domain are ( see Fig.1):

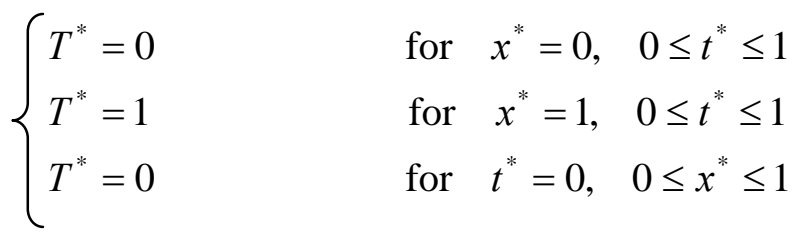

\section{Standard Galerkin finite element scheme}

After the discretization of the solution domain into a computational mesh (Fig.2), consisting of predetermined geometrical shapes, the prime unknowns in the governing equations are replaced by approximate forms defined within the selected finite elements. In the weighted residual finite element scheme, used in the present work, these unknowns are replaced by trial function representations, which in the context of a discretized domain are given by low order interpolation polynomials, $N_{j}[1]$ : 
$T^{*} \approx \tilde{T}^{*}=\sum_{j=1}^{n} N_{j} T_{j}^{*}$

where $n$ is total number of nodes in an element and, $T_{j}^{*}$ is the nodal values of unknown at the nodes (i.e. sampling points) of an element. Therefore, the above equation provides approximate values for unknown within an element via interpolation using its nodal values. Substitution of approximate values for the unknown from Eq. (7) into the governing Eq. (5), leads to the appearance of residual statements. These statements are then multiplied by appropriate weight functions $\left(w_{i}\right)$ and integrated over an element domain. Following the described step we obtain:

$\int_{\Omega_{e}}\left[W_{i}\left(\frac{\partial \sum_{j=1}^{n} N_{j} T_{j}^{*}}{\partial t^{*}}+C \frac{\partial \sum_{j=1}^{n} N_{j} T_{j}^{*}}{\partial x^{*}}-D \frac{\partial^{2} \sum_{j=1}^{n} N_{j} T_{j}^{*}}{\partial x^{* 2}}-f^{*}\right)^{*}\right] d x^{*} d t^{*}=0$

The second order differentials in Eq. (8) are reduced by the application of Green's theorem (i.e. generalised form of integration by parts). This leads to the appearance of boundary integral (flux) terms along the exterior boundaries of finite elements. For each interpolation function a weight function can be used to generate weighted residual equations such as Eq. (8). Therefore corresponding to a total of $n$ interpolation functions, $n$ equations are generated and a system of $n \times n$ equations is constructed. Using matrix notation this system is written as [25]:

$\left[A_{i j}\right]\left\{T_{j}^{*}\right\}=\left\{B_{j}\right\}$

where

$$
A_{i j}=\int_{\Omega_{e}}\left[W_{i}\left(\frac{\partial N_{j}}{\partial t^{*}}+D \frac{\partial N_{j}}{\partial x^{*}}\right)+C \frac{\partial W_{i}}{\partial x^{*}} \frac{\partial N_{j}}{\partial x^{*}}\right] d x^{*} d t^{*}
$$


$B_{j}=\int_{\Omega_{e}} W_{i} f^{*} d x^{*} d t^{*}+\int_{\Gamma_{e}} W_{i} \frac{\partial T^{* e}}{\partial x^{*}} n_{x} d \Gamma_{e}$

A system of weighted residual equations should be derived for each element in the domain. This is obviously not convenient. However, by using an elemental coordinate system rather than the global coordinates the uniformity of the matrix Eq. (9) can be preserved. This is achieved using isoparametric mapping of elements of the global mesh into a master element where all the calculations are carried out [1]. In addition, a natural coordinate system such as $-1 \leq \xi, \tau \leq+1$ can be used within the master element to enable the evaluation of all integrals within its domain by Gauss quadrature method [26].

\section{The $\theta$ time stepping method}

In this method, initially, any time derivatives in the governing equations are kept unchanged whilst the spatial discretization is carried out. At the end of this process a system of ordinary differential equations in terms of time derivatives is generated. The following time stepping is then applied to this system.

For a class of single step theta methods $(0 \leq \theta \leq 1)$ this system can be written in matrix form as [25]:

$$
[M]_{\theta}\left\{\dot{T}^{*}\right\}+_{\theta}[A]_{\theta}\left\{T^{*}\right\}_{\theta}=\{B\}_{\theta}
$$

Where the subscript $\theta$ indicates that the weighted residual statement is derived at time level $\theta$ and $M$ is mass matrix. If time derivative is written as:

$\left\{T^{*}\right\}=\frac{\left\{T^{*}\right\}_{n+\Delta t}-\left\{T^{*}\right\}_{n}}{\theta \Delta t^{*}}=\frac{\left\{T^{*}\right\}_{n}-\left\{T^{*}\right\}_{n}}{\Delta t^{*}}$

Using above equations and after some algebraic manipulation we have [25]:

$$
\begin{array}{r}
\left([M]_{\theta}+\theta \Delta t^{*}[A]_{n+1}\right)\left\{T^{*}\right\}_{n+1}=\left([M]_{\theta}+(1-\theta) \Delta t^{*}[A]_{n 1}\right)\left\{T^{*}\right\}_{n}+ \\
\left((1-\theta)\{B\}_{n}+\theta\{B\}_{n+1}\right) \Delta t^{*}
\end{array}
$$

\section{Multiscale finite element modelling}

In dealing with transient transport problems formulated in terms of previously described governing equations multiscale behaviour with respect to both space and time variables 
can be expected. The following approach which is the extension of the method developed by Parvazinia et al. [16] for steady state problems yields stable solutions.

\section{1 variational multiscale method using bubble functions}

Let us consider a problem defined in $\Omega \subset \mathrm{R}^{2}$ as

$$
\begin{cases}L(T)=f & \text { in } \Omega \\ T=0 & \text { on } \Gamma\end{cases}
$$

where $L$ is differential operator, which includes both temporal and spatial components, and $f$ is a given source function defined in $\Omega$ [14]. Here the time dependent convectiondiffusion operator can be written as:

$L=\frac{\partial}{\partial t^{*}}+C \nabla-D \Delta$

The standard Galerkin method is formulated in a subspace $\mathrm{V}_{\mathrm{h}} \subset \mathrm{V}$, where $\mathrm{V}$ is the space of functions for which a solution of the continuous problem is sought. The Galerkin method aims to find $T_{\mathrm{h}} \in \mathrm{V}_{\mathrm{h}}$ such that

$a\left(T_{h}, v\right)=\left(L T_{h}, v\right)=(f, v)$

where $\mathrm{a}(.,$.$) is a bilinear form and (.,$.$) representing the scalar product of its$ arguments. In a two-scale method, the unknowns are divided into two parts

$\left\{\begin{array}{l}T_{h}=T_{1}+T_{b} \\ v_{h}=v_{1}+v_{b}\end{array}\right.$

where $T_{b}$ is the fine scale and $T_{1}$ represents a standard finite element approximation polynomial (interpolation function). The fact that bubble functions disappear on element boundaries[17-24] makes it possible to remove the equations that correspond to these functions from the set of elemental equations. This procedure is called static condensation [27]. In the static condensation procedure we set $v=v_{b}$ in Eq. (14) to obtain:

The variational formulation may be written as [14,15]:

$$
a\left(v_{h}, T_{h}\right)=\left(v_{h}, f\right) \text { or } a\left(v_{1}+v_{b}, T_{1}+T_{b}\right)=\left(v_{1}+v_{b}, f\right)
$$


it can be written as two sub-problems

$$
\begin{aligned}
& a\left(v_{b}, T_{1}\right)+a\left(v_{b}, T_{b}\right)=\left(v_{b}, f\right) \\
& a\left(v_{1}, T_{1}\right)+a\left(v_{1}, T_{b}\right)=\left(v_{1}, f\right) .
\end{aligned}
$$

Under a steady state condition the transport field behaviour is the same in all directions and a general elemental bubble function can have the same coefficient in all directions. In a transient problem where the spatial and temporal behaviours are different using a general elemental bubble function may become impractical. This problems can be resolved by separating spatial bubble functions from temporal bubble functions as

$$
\left\{\begin{array}{l}
T_{b}=T_{b s}+T_{b t} \\
v_{b}=v_{b s}+v_{b t}
\end{array}\right.
$$

Therefore Eq. (18) can be rewritten as:

$$
a\left(v_{b s}+v_{b t}, T_{1}\right)+a\left(v_{b s}+v_{b t}, T_{b s}+T_{b t}\right)=\left(v_{b s}+v_{b t}, f\right)
$$

The above equation can be written as two sub-problems

$$
\begin{aligned}
& a\left(v_{b s}, T_{1}\right)+a\left(v_{b s}, T_{b s}\right)+a\left(v_{b s}, T_{b t}\right)=\left(v_{b s}, f\right) \\
& a\left(v_{b t}, T_{1}\right)+a\left(v_{b t}, T_{b s}\right)+a\left(v_{b t}, T_{b t}\right)=\left(v_{b t}, f\right)
\end{aligned}
$$

The above equations imply that for spatial and temporal directions static condensation is done separately.

Using bubble functions in $x$ and $t$ directions the bubble enriched Lagrangian shape functions in local coordinate system $\xi(-1,+1), \tau(-1,+1)$ can be written as:

$$
\left\{\begin{array}{l}
N_{1}=\frac{1}{4}(1-\xi)(1-\tau)+b \phi_{\xi}+b t \phi_{\tau} \\
N_{2}=\frac{1}{4}(1+\xi)(1-\tau)-b \phi_{\xi}+b t \phi_{\tau} \\
N_{3}=\frac{1}{4}(1+\xi)(1+\tau)+b \phi_{\xi}+b t \phi_{\tau} \\
N_{4}=\frac{1}{4}(1-\xi)(1+\tau)-b \phi_{\xi}+b t \phi_{\tau}
\end{array}\right.
$$


Where $\phi_{\tau}$ and $\phi_{\xi}$ are the temporal and spatial bubble functions, respectively, (i.e. the functions that are used to enrich the normal Lagrangian shape functions) and $b$ is an adjustable parameter called the bubble coefficient. The methods used for the determination coefficient $b$, via the static condensation, and the spatial bubble function $\phi_{\xi}$ have been published previously and will not be discussed here [16]. In the present work the effects of bubble coefficient adjustment on the overall results are studied and shown in the results section. The following two types of bubble functions for temporal discretization are used:

$$
\begin{aligned}
& \phi_{\tau}=\sum_{q=1}^{n}\left(1-\tau^{2}\right)^{q} \\
& \phi_{\tau}=\sum_{q=1}^{n}\left(1-\tau^{2 q}\right)
\end{aligned}
$$

For $q=1$ the bubble is $2^{\text {nd }}$ order, $q=2$ implies that the bubble is $4^{\text {th }}$ order and so on.

\subsection{Elimination of the boundary integrals}

In discretizations involving bubble functions we can not assume that inter-element boundary integrals will be automatically eliminated during the assembly of elemental equations. This problem does not become apparent in the one dimensional case as the boundary integrals are reduced to simple nodal flux terms. The variational formulation for the transient convection-diffusion equation, after application of Green's theorem is

$$
\left(\frac{\partial T_{h}}{\partial t}, v\right)+\left(C \nabla T_{h}, v\right)+\left(D \nabla T_{h}, \nabla v\right)=(f, v)
$$

Substitution from Eq. (19) gives:

$$
\left(\frac{\partial T_{h}}{\partial t}, v\right)+\left(C \nabla T_{h}, v\right)+\left(D \nabla T_{1}, \nabla v\right)+\left(D \nabla T_{b}, \nabla v\right)=(f, v)
$$

If $v$ is a linear test function (weight function) according to Green's theorem [28] we have:

$$
(\nabla v, \nabla \phi)_{\Omega_{e}}=-(\Delta v, \phi)_{\Omega_{e}}+(\nabla v, \phi)_{\Gamma_{e}}=0
$$

where $\phi$ is bubble function. Therefore the last term in RHS of Eq. (27) can be written as: 
$\left(\nabla v, D \nabla T_{b}\right)_{\Omega_{e}}=-\left(\Delta v, D T_{b}\right)_{\Omega_{e}}+\left(\nabla v, D T_{b}\right)_{\Gamma_{e}}=0$

Hence Eq. (27) is reduced to:

$\left(\frac{\partial T_{h}}{\partial t}, v\right)+\left(C \nabla T_{h}, v\right)+\left(D \nabla T_{1}, \nabla v\right)=(f, v)$

As can be seen the bubble function does not affect the Laplacian term and therefore no boundary integral due to the bubble function exists.

\section{Analytical solution of the governing equations}

To validate the numerical solutions, the following analytical solutions of the dimensionless equations are used presented. These solutions are found via the Laplace transform method.

Transient diffusion problem:

$T^{*}\left(x^{*}, t^{*}\right)=\frac{2}{\pi} \sum_{n=1}^{\infty} \frac{(-1)^{n}}{n} \exp \left(\frac{-n^{2} \pi^{2} D t^{*}}{l}\right) \sin \left(\frac{n \pi x^{*}}{l}\right)+\frac{x^{*}}{l}$

Transient convection-diffusion problem for $D=1$ (in all simulations $D=1$ and $C$ is adjusted):

$$
\begin{array}{r}
T^{*}\left(x^{*}, t^{*}\right)=\exp \left(0.5 \frac{C}{D}\left(x^{*}-l\right)\right) \sum_{n=1}^{\infty} \frac{2 \pi}{n^{2} \pi^{2}+0.25\left(\frac{C}{D}\right)^{2}} n(-1)^{n} \\
\exp \left(\frac{-\left(n^{2} \pi^{2}+0.25\left(\frac{C}{D}\right)^{2}\right) t^{*}}{l}\right) \sin \left(\frac{n \pi x^{*}}{l}\right)
\end{array}
$$

where $l$ is the domain length in $x$ direction. For dimensionless problem $l=1$.

\section{Results and discussion}

The main objective of the present work has been the construction of a new scheme for the space-time approximation of field unknowns in Galerkin finite element method. Analytical solutions are obtained for bench mark problems to validate the numerical results. In this section the two sets of results are compared to evaluate the ability of the scheme to generate theoretically expected simulations. In addition numerical results obtained using the theta time stepping method are also compared with proposed model 
results. An in house developed computer code has been used to solve the transient diffusion and convection-diffusion problems via the present scheme. In all of the figures shown in this section $\mathrm{b}$ and bt represent the bubble coefficients and $l_{\mathrm{x}}$ and $l_{\mathrm{t}}$ indicate the element length in $x$ and $t$ directions, respectively.

Figs. 1 and 2 show the solution domain, its boundaries and the finite element meshes used to obtain the numerical results of the benchmark problems. As stated previously identical temporal and spatial discretizations are used to construct the required finite element approximations. Three different mesh schemes are used. In all of the numerical experiments carried out the coarse scale variations are approximated by linear Lagrangian shape functions in the Galerkin finite element scheme. It is shown that despite using a coarse mesh, bubble enriched shape functions can provide stable-accurate solutions for multiscale problems.

Figs 3-13 show the results for the transient diffusion problem. For $D=1$ and $l_{\mathrm{t}}=0.1$ and 0.02 the transient solution is unstable while with $l_{\mathrm{t}}=0.002$ the accurate-stable solution can be obtained. The instability shown in Fig. 3 demonstrates the multiscale nature of the problems in this case. However, the solution is stabilised after the utilization of the bubble function based scheme. Fig.7 shows that at $D=5$ although multiscale behaviour increases using $l_{\mathrm{t}}=0.002$ (i.e. very refined mesh) an accurate and stable solution can be generated. To avoid excessive mesh refinement for $l_{t}=0.1$ and 0.02 bubble functions are applied. Figs 8 and 9 show the results obtained using two types of bubble functions based on the Eqs.24 and 25, respectively. Fig.9 indicates that the bubble represented in the Eq. 25 has a better performance under a range of conditions ( here the $4^{\text {th }}$ order bubble function of this type is used). The theta method based on identical time step also yields an accurate solution at $\Delta t^{*}=0.002$ (Fig.10). Although by increasing diffusion coefficient to $D=10$ the multiscale behaviour increases the bubble functions can still generate stable solution. For mesh scheme $2\left(l_{\mathrm{t}}=0.02\right)$, as Fig. 12 shows, a stable solution can also be obtained at $b t=2$. It must be noted that while the problem does not demonstrate any spatial multiscale behaviour in this case, the temporal behaviour is strongly multiscale in nature. 
Figs. 14-23 show the results for the transient convection-diffusion equation. In this case in both temporal and spatial dimensions multiscale behaviour may be observed. At $C=5$ using the refined mesh scheme $3\left(l_{\mathrm{t}}=0.002\right)$ a stable solution is obtained (Fig. 14) which is similar to the results generated by the theta method with $\Delta t^{*}=0.002$ (Fig. 15). At $C=10$, as Fig. 17 shows, mesh schemes 1and 2 can yield stable solutions only with bubble enriched elements while the more refined mesh scheme 3 gives stable-accurate results with ordinary elements. This shows that the temporal disretization used in mesh scheme 3 is fine enough to over come the multiscale behaviour. Fig. 18 shows the corresponding results generated by the theta method. Although the stable solution can be achieved by theta method at $C=10$ the solution is slightly underestimated in comparison to the exact solution. At $C=50$, as Fig. 19 shows, even using the mesh scheme 3 the solution is slightly unstable and over shoots the analytical result. In this case using bubble function approach stable solutions are obtained (Figs. 19 and 20). As Fig. 20 shows with theta method the solution is slightly under estimated. Therefore at higher convection coefficients of $C=10$ and 50 the theta method generates stable results but they are not very accurate. It must be noted that since at $C=50$ the exact solution at $\mathrm{x}^{*}=0.9$ is nearly zero (the cross section $\mathrm{x}^{*}=0.9$ is used in all numerical experiments to show the solution in temporal direction) an over-diffusive multiscale solution is intentionally used to show the results in $t^{*}$ direction (see Fig. 21).

Considering Figs. 22 and 23, different temporal and spatial multiscale behaviour can be observed. As Fig. 22 shows at $C=10$ and $l_{\mathrm{x}}=0.1$ in the spatial dimension the solution is stable and very close to the exact solution, however, it is distinctly unstable in temporal dimension (mesh scheme 1). As Fig. 23 shows even with $l_{x}=0.02$ ( mesh scheme 2) the solution still remains unstable. These results confirm that the behaviour in the temporal dimension is extremely multiscale. Comparison of Figs. 17 and 19 shows that when the convection coefficient is increased from $C=10$ to $C=50$ a corresponding increase in the bubble coefficient stabilizes the solution. Therefore, if the bubble coefficient is treated as a measure of the level of multiscale behaviour it is seen that at $C=50$ this coefficient in $x$ direction is $\mathrm{b}=0.45$ (Fig. 21) whilst in $t$ direction (using the same level of discretization as mesh scheme 1) it is bt=20 (Fig. 20). This clearly shows the difference in the level of multiscale behaviour in spatial and temporal dimensions. 
The causes of highly multiscale behaviour with respect to the time variable can be found in the analytical solution. In the pure diffusion problems if no spatial multiscale behaviour exists, the transient response is determined byexp( $\left.\frac{-n^{2} \pi^{2} D t^{*}}{l}\right)$. The exponential argument becomes large for small values of $D$ thus becoming a source of strong multiscale behaviour and temporal instability. A similar situation arises in convection-diffusion problems. The spatial behaviour is affected by $\exp \left(0.5 \frac{C}{D}\left(x^{*}-l\right)\right)$ and while the temporal behaviour is affected by $\exp \left(\frac{-\left(n^{2} \pi^{2}+0.25\left(\frac{C}{D}\right)^{2}\right) t^{*}}{l}\right)$ in which $(C / D)^{2}$ become large at smaller values of $(C / D)$ and therefore, strong temporal multiscale behaviour is observed. In both cases, however, when $l$ is small the exponential arguments are large. In finite element disretization $l=l_{\mathrm{x}}$ using finer meshes with respect to $x$ the solution shows temporal instability (stronger temporal multiscale behaviour). As Fig. 25 shows using mesh scheme $2\left(l_{\mathrm{x}}=0.1, l_{\mathrm{t}}=0.02\right)$ the solution with $b t=2.5$ is unstable while for mesh scheme $4\left(l_{\mathrm{x}}=0.2, l_{\mathrm{t}}=0.02\right)$ with the same temporal discretization the solution remains stable. This is why the standard Galerkin finite element solution of transient convection-diffusion equation with traditional time stepping methods can only have conditional stability [29]. Such stability conditions can be derived by relating $\frac{\Delta x^{*}}{\Delta t^{*}}$ to the eigen values of the stiffness matrix.

\section{Conclusion}

A series of numerical experiments validated through comparison with their corresponding analytical solutions are used to evaluate the performance and efficiency of a proposed space-time bubble enriched discretization scheme. The numerical results are also compared with the conventional data obtained via theta time stepping method. The proposed bubble enriched variational multiscale method is shown to be capable of generating stable accurate results for transient diffusion and convection-diffusion equations. Although the theta time stepping method gives stable solutions but, by 
increasing the transport coefficients ( $D$ or $C$ ) the time intervals must be decreased and computational cost increases dramatically. In the proposed multiscale method since the scheme uses the same discretization in both temporal and spatial dimensions, the unsteady problem is solved similar to a steady problem making the method particularly cost effective.

\section{References:}

[1] O.C.Zienkiewicz and R.L.Taylor, The finite element method, McGraw-Hill, London, 1994.

[2] J.Donea, A Taylor-Galerkin method for convective transport problems, International Journal for Numerical Methods in Engineering. 20 (1984) 101-120.

[3] J.Donea, B.Roig, and A.Huerta, High order accurate time stepping schemes for convection-diffusion equation. Computer methods in applied mechanics and engineering 182 (2000) 249-275.

[4] B.C.Bell and K.S.Surana A space-time coupled p-version least squares finite element formulation for unsteady two-dimensional Navier-Stokes equations, international journal for numerical methods in engineering, 39 (1969) 2593-2618.

[5] J.T. Oden, A general theory of finite element, Part I and II', international journal for numerical methods in engineering, 1 (1969) 205-221,247-259

[6] J.H. Argyris, and D.W.Scharpf, Finite elements in time and space, nuclear engineering and design, 10 (1969) 456-464.

[7] T.J.R.Hughes, and G.M.Hulbert, Space-time finite element methods for elastodynamics: fomulations and error estimates, Computer Methods in Applied Mechanics and Engineering, 66 (1998) 339-363.

[8] S.Mittal, and T.E.Tezduyar, Notes on the stabilized space-time finite-element formulation of unsteady incompressible flows, Computer Physics Communications 73 (1992) 93-112.

[9] T.E.Tezduyar, and M.Behr, A new strategy for finite element computations 
involving moving boundaries and interfaces -The deforming-spatial-domain / space-time procedure: I. The concept and the preliminary numerical tests, Computer Methods in Applied Mechanics and Engineering 94 (1992) 339-351

[10] D.A.French, A space-time finite element method for the wave equation, Computer Methods in Applied Mechanics and Engineering 107 (1993) 145-157.

[11] C.L.Bottasso, On the computation of the boundary integral of space-time deforming finite elements, Communications in numerical methods in engineering, 13 (1997) 53-59.

[12] E.Onate, and M.Manzan, A general procedure for deriving stabilized space-time finite element methods for advective-diffusive problems, international journal for numerical methods in engineering, 31 (1999) 203-221.

[13] J.Réthoré, A.Gravouil, and A.Combescure, A combined space-time extended finite element method, international journal for numerical methods in engineering, 64 (2005) 260-284.

[14] T.J.R.Hughes, and J.Stewart, A space time formulation for multiscale phenomena. Journal of computational and applied mathematics. 74 (1996) 217-229.

[15] T.J.R.Hughes, Multiscale phenomena, Green’s functions, the Dirichlet - toNeumann formulation, subgrid scale models, bubbles and the origins of stabilized methods. Computer methods in applied mechanics and engineering 127 (1995) 381401.

[16] M.Parvazinia, V.Nassehi and R.J.Wakeman, Multiscale finite element modelling of laminar steady flow through highly permeable porous media, Chemical Engineering Science, 61 (2006) 586-596.

[17] F.Brezzi, M.Bristeau, L.P.Franca, M.Mallet, M. and G.Roge, A relationship between stablized finite element methods and the Galerkin method with bubble functions. Computer methods in applied mechanics and engineering 96 (1992) 117129.

[18] F.Brezzi, L.P.Franca, T.J.R. Hughes, and A.Russo, $b=\int g$. Computer methods in applied mechanics and engineering 145 (1997) 392-339. 
[19] C.Baiocchi, F. Brezzi, and L.P.Franca, Virtual bubbles and Galerkin-least-squares type methods. Computer methods in applied mechanics and engineering 105 (1993) 125-141.

[20] L.P.Franca, T.J.R. Hughes and R.Stenberg, Stabilized finite element methods for the Stokes problem. In: Nicolaides, R.A. and Gunzberger, M.D. (Eds.), Incompressible Fluid Dynamics-Trends and Advances, Cambridge University Press, Cambridge, 1993.

[21] L.P.Franca, C. Farhat, A.P. Macedo and M.Lesoinne, Residual free bubbles for Helemholtz equation. Computer methods in applied mechanics and engineering 40 (1997) 4003-4009.

[22] L.P.Franca, and A.Russo, Deriving upwinding, mass lumping and selective reduced integration by residual free bubbles, Applied Mathematics Letters 9 (1996) 83-88.

[23] L.P.Franca and A.Russo, Unlocking with residual-free bubbles. Computer methods in applied mechanics and engineering 142 (1997) 361-364.

[24] L.P.Franca and A.Russo, Mass lumping enamating from residual free bubbles. Computer methods in applied mechanics and engineering 142 (1997) 353-360.

[25] V.Nassehi, Practical aspects of finite element modelling of polymer processing, John Wiely \& Sons, Chichester, 2002.

[26] C.F.Gerald and P.O.Wheatley, 1984. Applied numerical analysis, Addison-Wesley.

[27] K.J.Bathe, Finite Element Procedures, Prentice Hall, Englewood Cliffs, N.J., 1996.

[28] L.P.Franca and C.Farhat, Bubble functions prompt unusual stabilized finite element methods, Computer methods in applied mechanics and engineering 123 ( 1995) 299-308.

[29] V.Nassehi, Numerical modelling of tidal dynamics in a one-dimensional branching estuary, PhD thesis, University of Wales, Swansea, UK, 1981. 


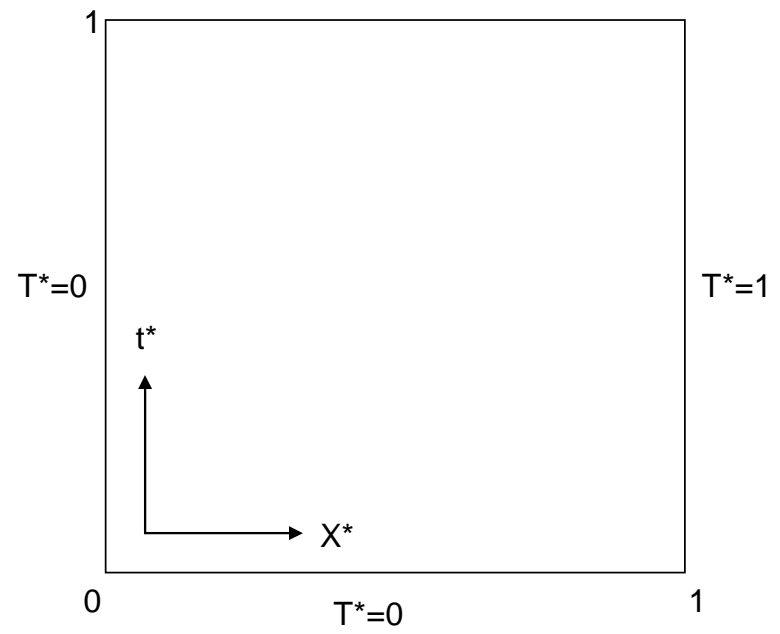

Fig.1. Domain ant its boundaries.

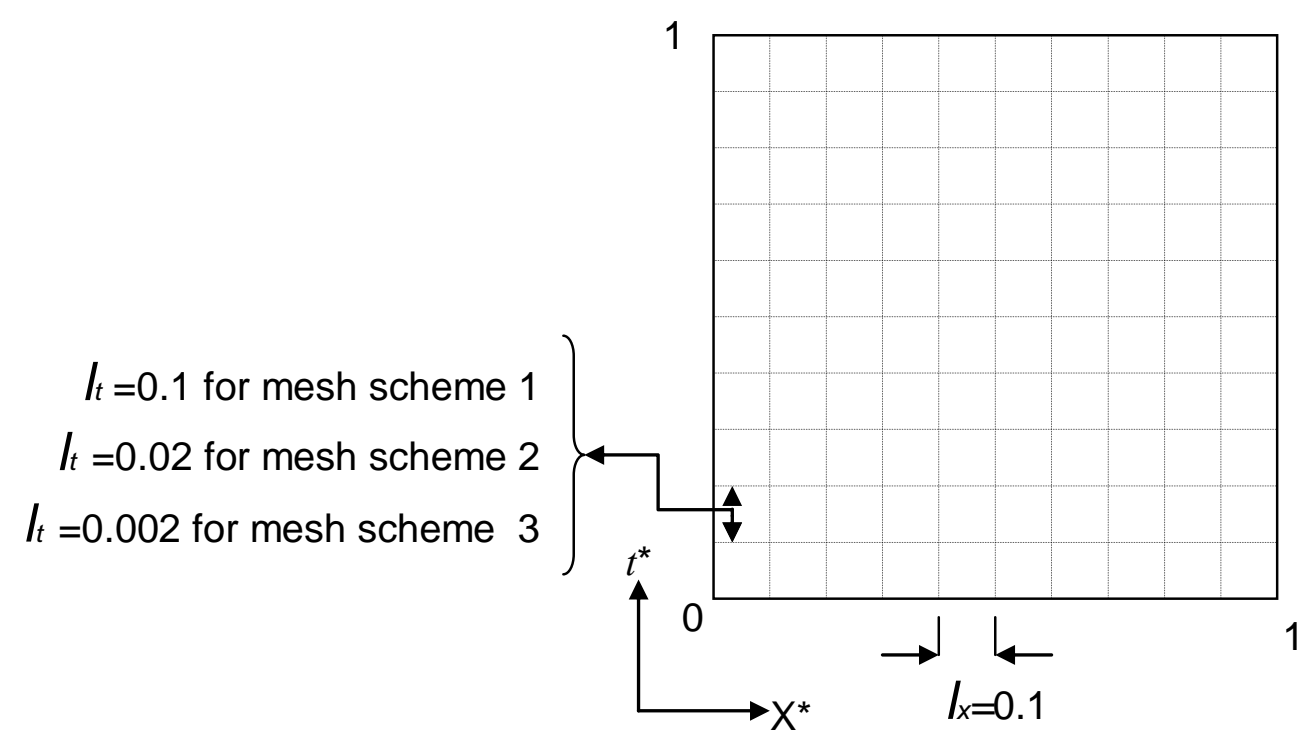

Fig.2. Finite element mesh schemes. 


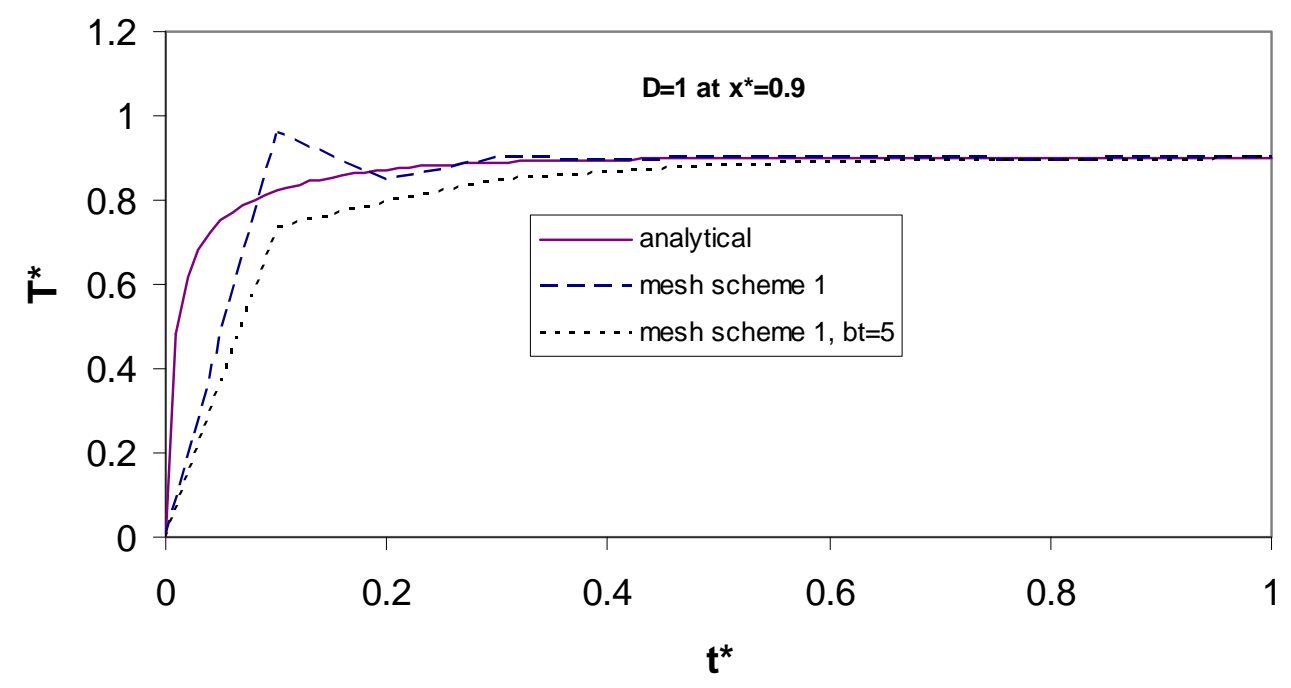

Fig. 3. Transient response of diffusion equation at $D=1$ and $x^{*}=0.9$. Mesh scheme 1 with and without temporal bubble function..

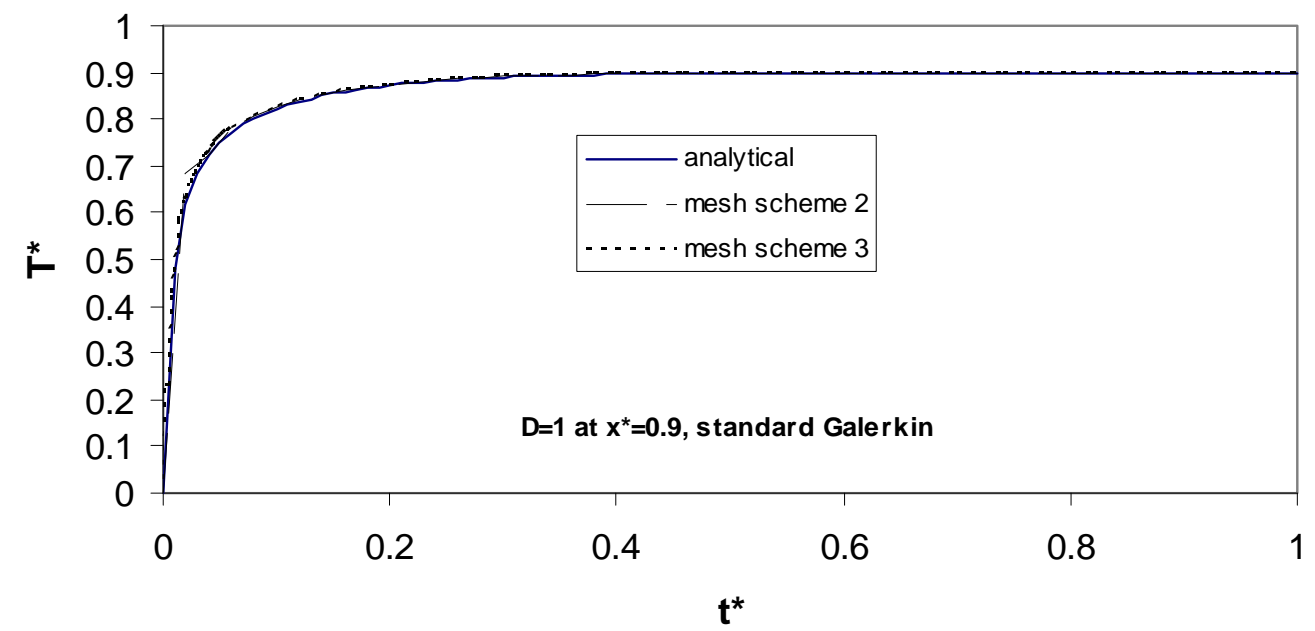

Fig. 4. Transient response of diffusion equation at $D=1$ and $x^{*}=0.9$. Mesh schemes 2 and 3. 


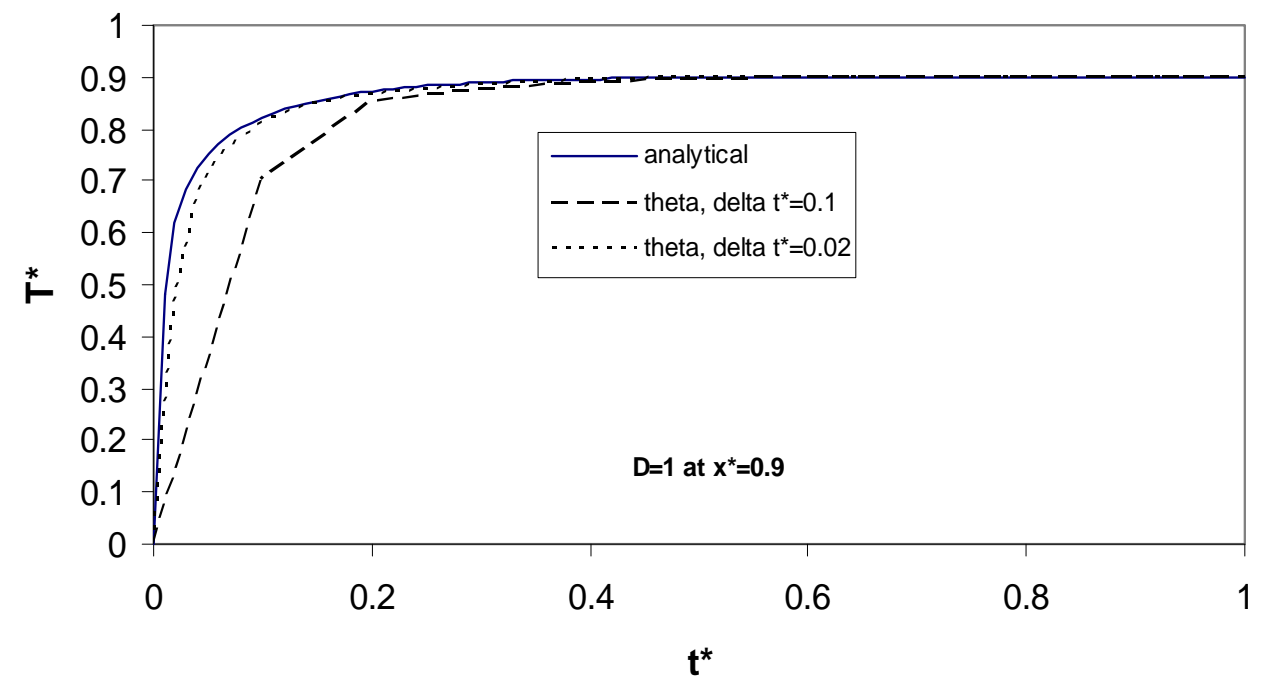

Fig. 5. Transient response of diffusion equation using theta method at $D=1$ and $\mathrm{x}^{*}=0.9$.

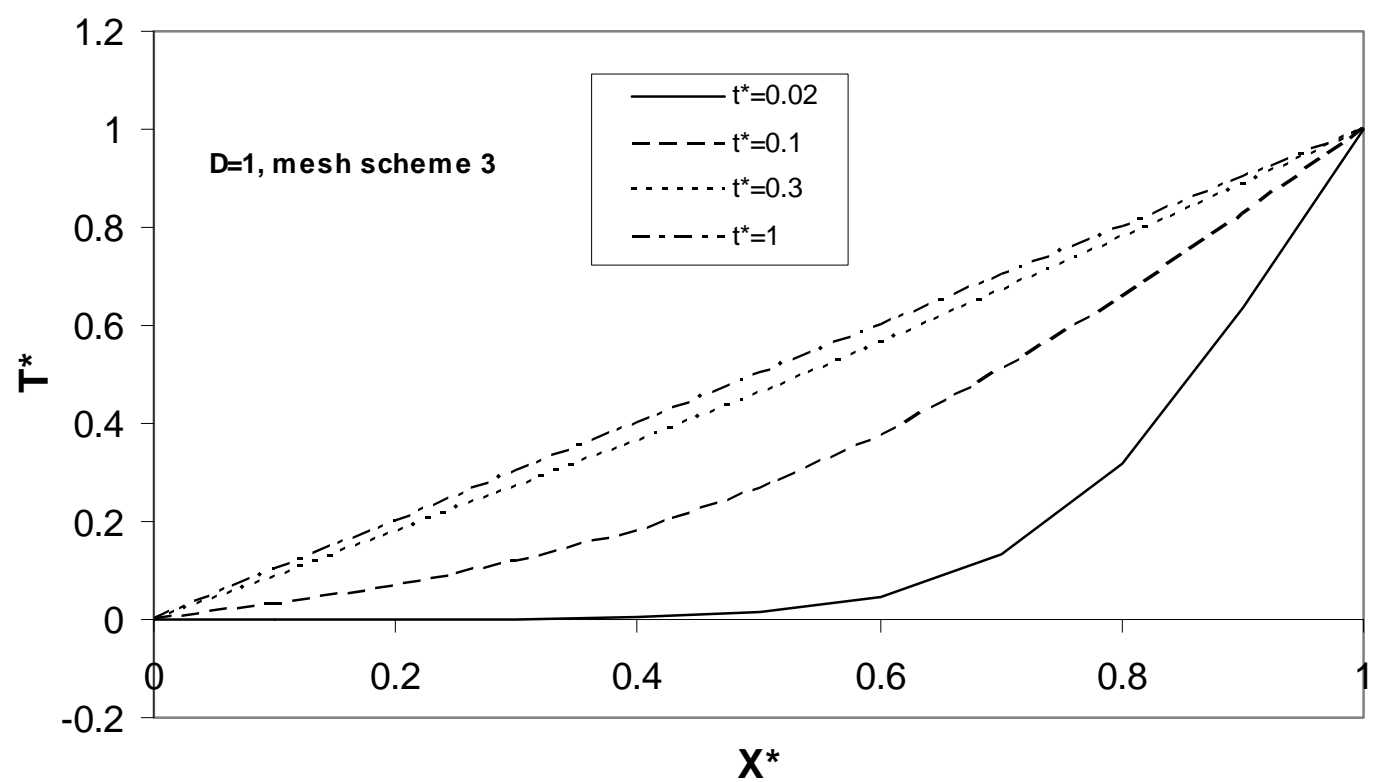

Fig. 6. Response of diffusion equation at different time sections for $D=1$ and $x^{*}=0.9$. mesh scheme 3 . 


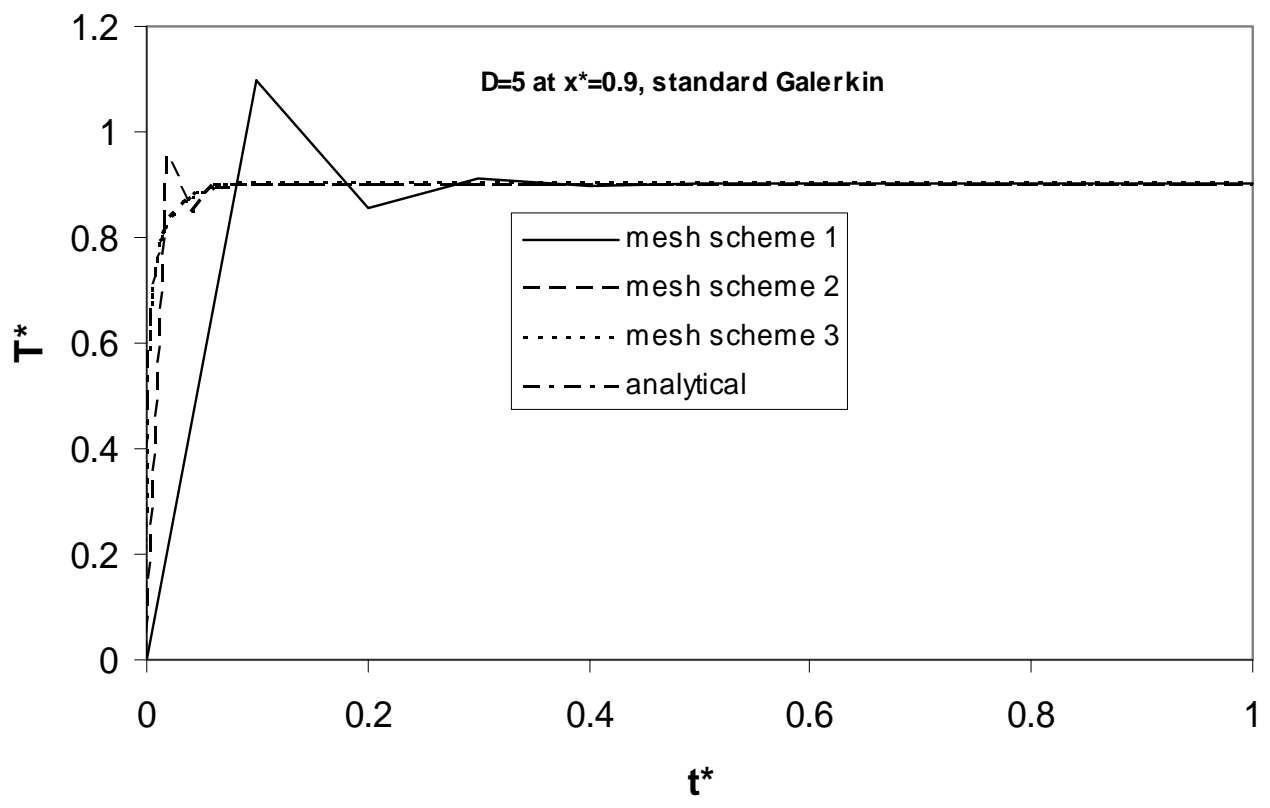

Fig. 7. Transient response of diffusion equation at $D=5$ and $x^{*}=0.9$ for all mesh schemes.

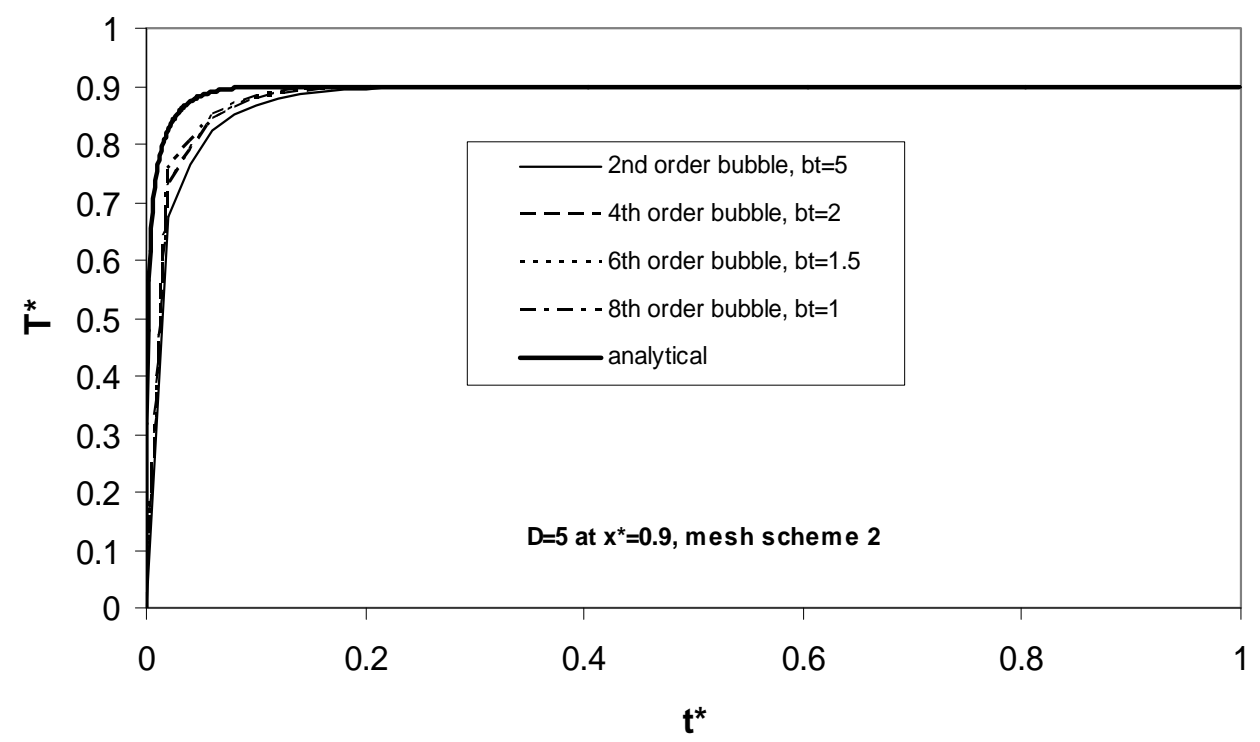

Fig. 8. Transient response of diffusion equation at $D=5$ and $x^{*}=0.9$ using different orders of the temporal bubble functions of Eq.24. 


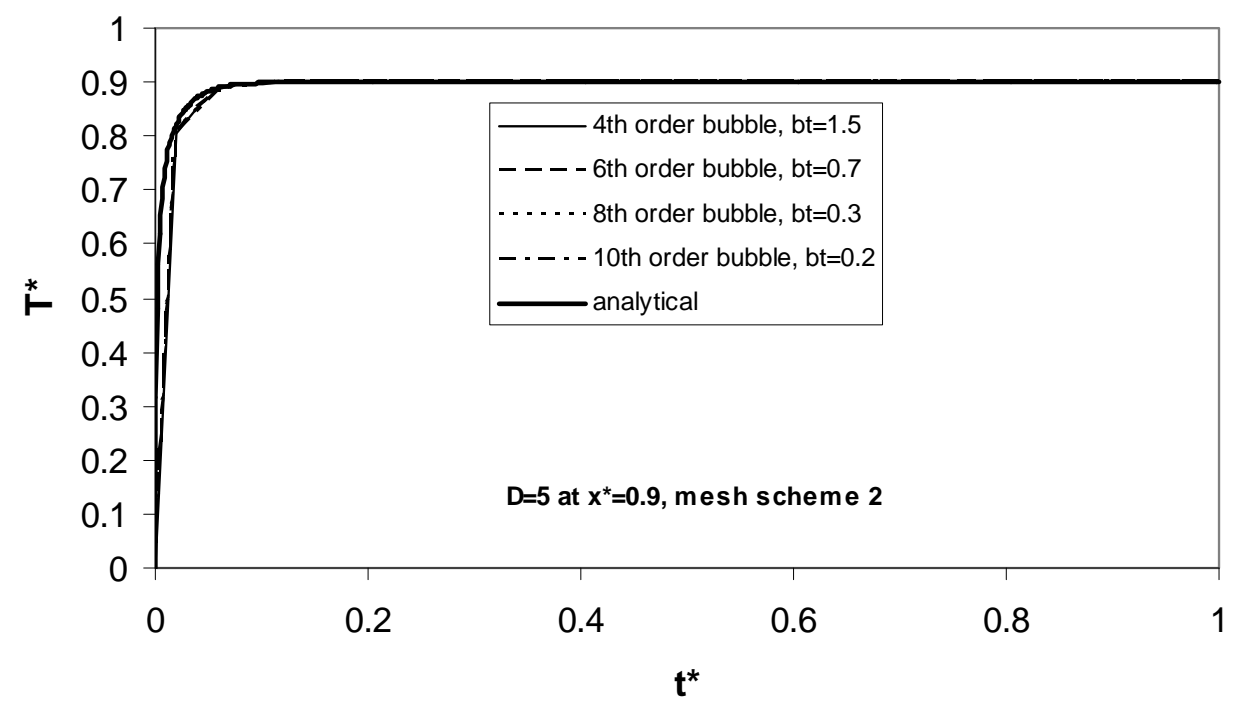

Fig. 9. Transient response of diffusion equation at $D=5$ and $\mathrm{x}^{*}=0.9$ using different orders of the temporal bubble functions of Eq.25.

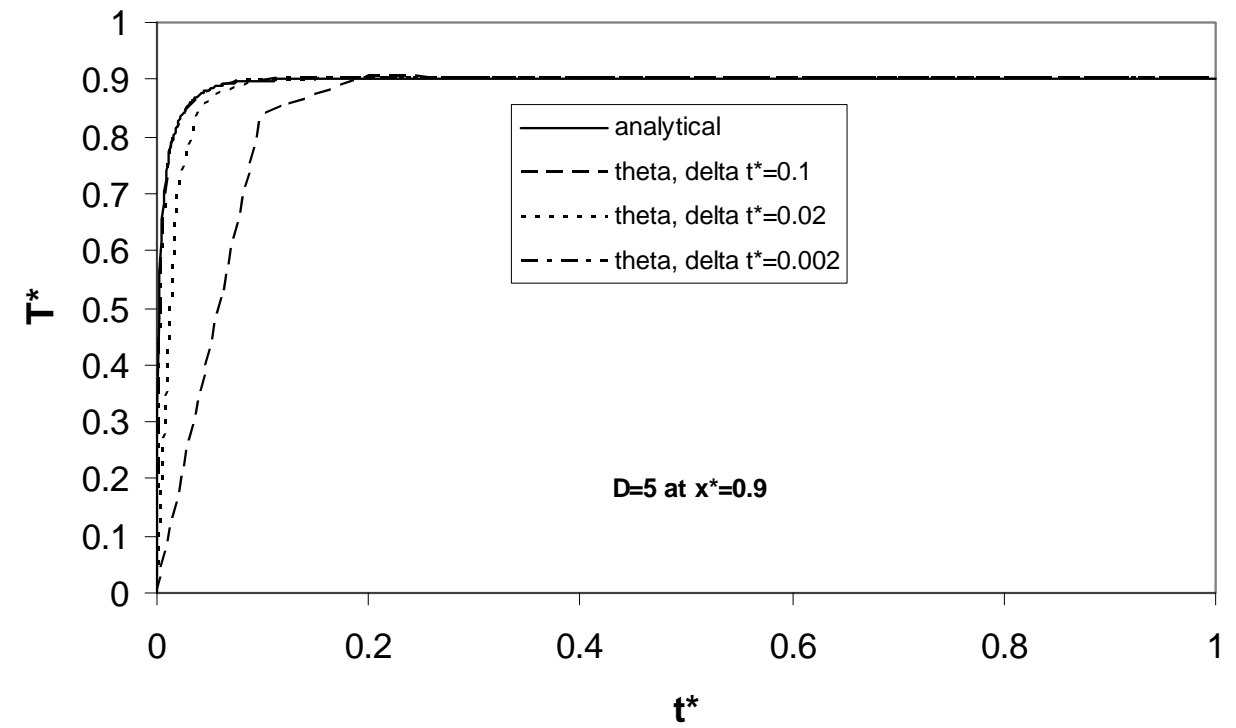

Fig. 10. Transient response of diffusion equation using theta method at $D=5$ and $\mathrm{x}^{*}=0.9$. 


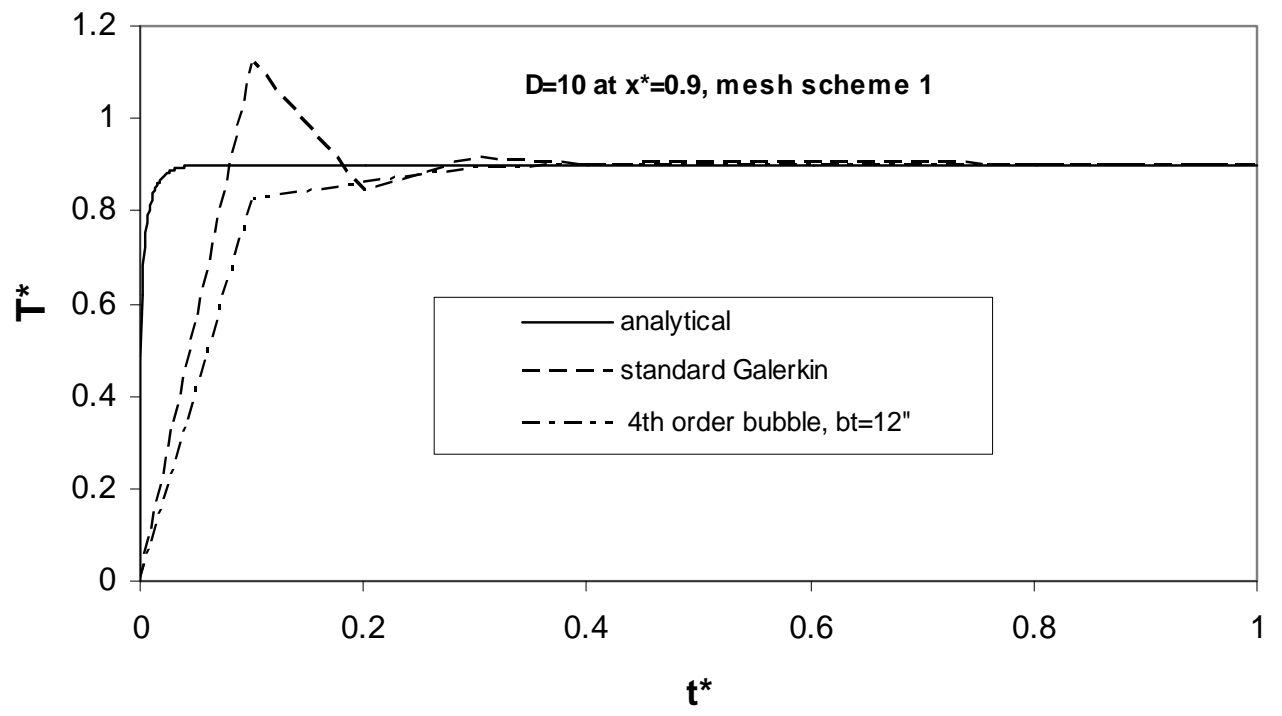

Fig. 11. Transient response of diffusion equation at $D=10$ and $x^{*}=0.9$. Mesh scheme 1 with and without temporal bubble function.

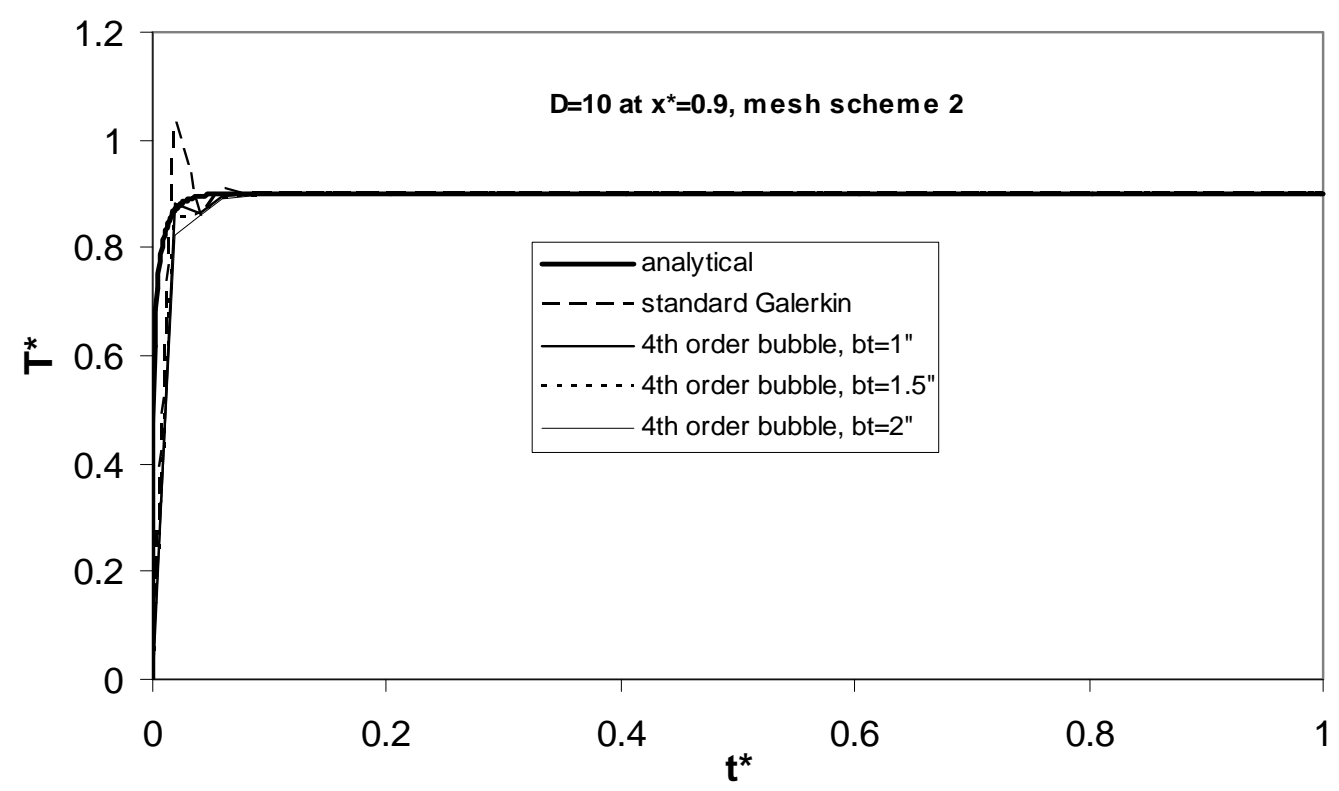

Fig. 12. Transient response of diffusion equation at $D=10$ and $\mathrm{x}^{*}=0.9$. Mesh scheme 2 with and without temporal bubble. 


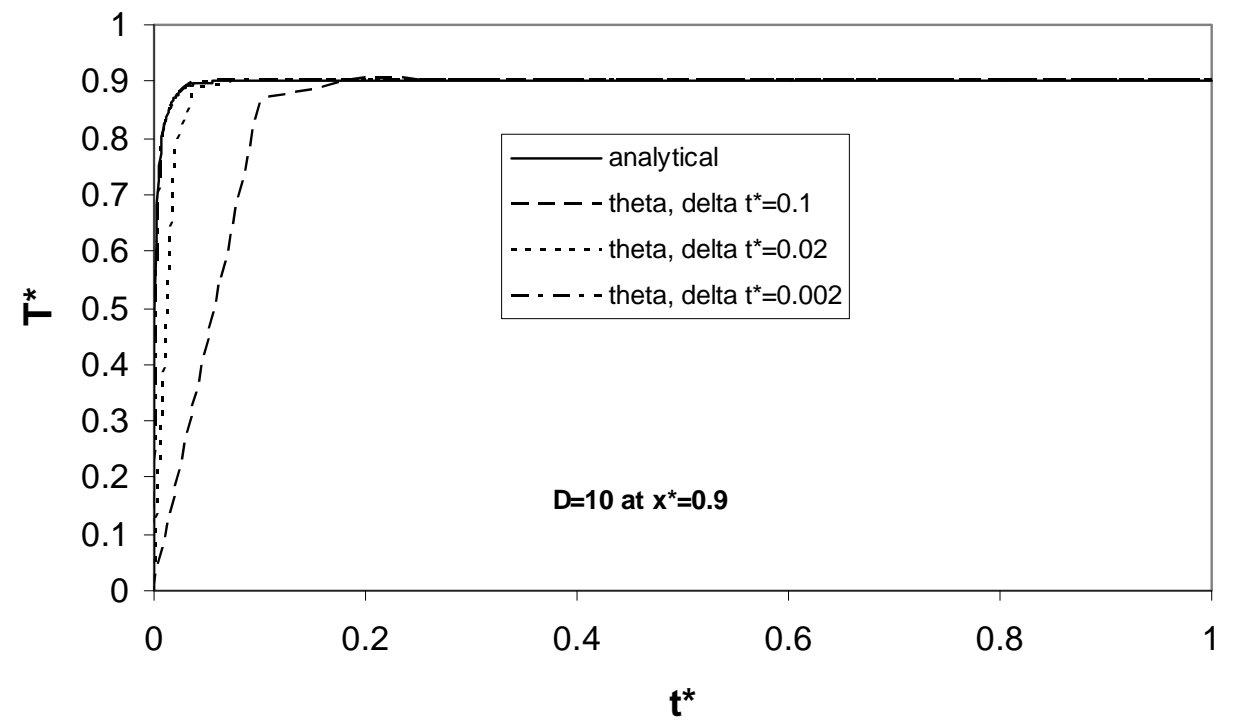

Fig. 13. Transient response of diffusion equation using theta method at $D=10$ and $\mathrm{x}^{*}=0.9$.

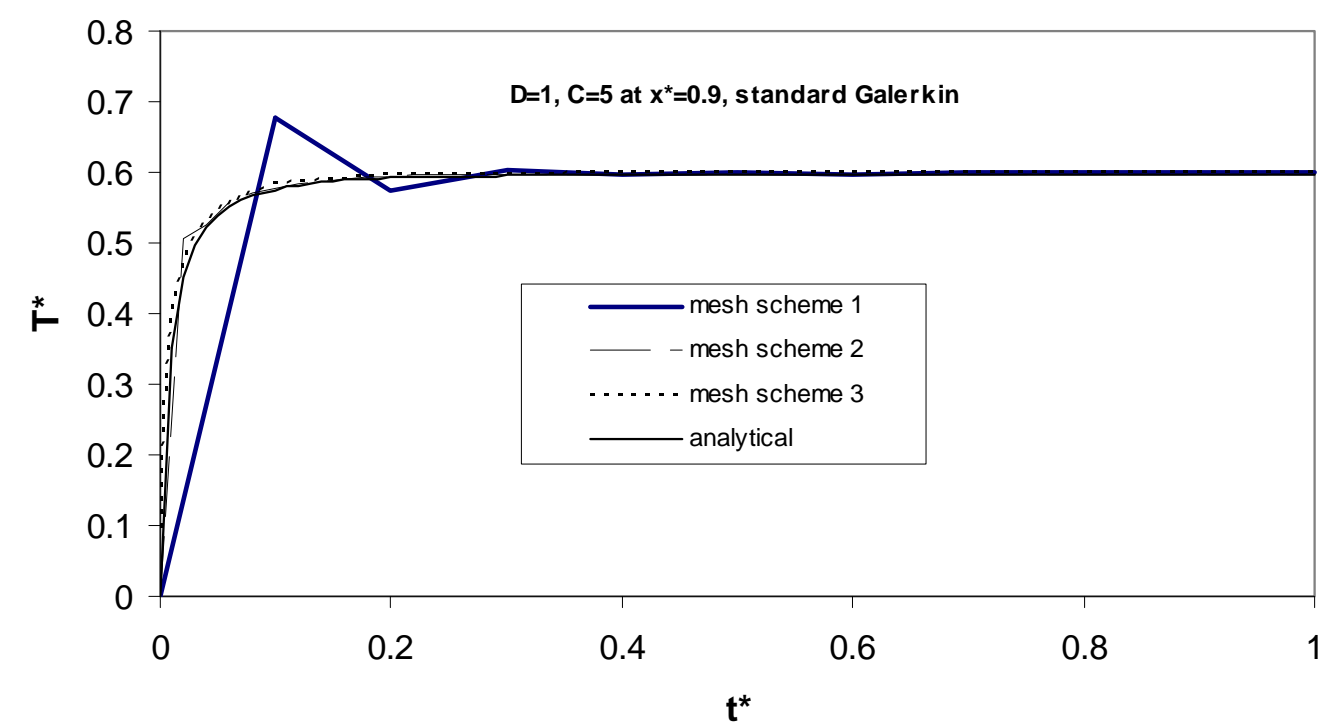

Fig. 14. Transient response of convection-diffusion equation at $D=1, C=5$ and $\mathrm{x}^{*}=0.9$ for all mesh scheme. 


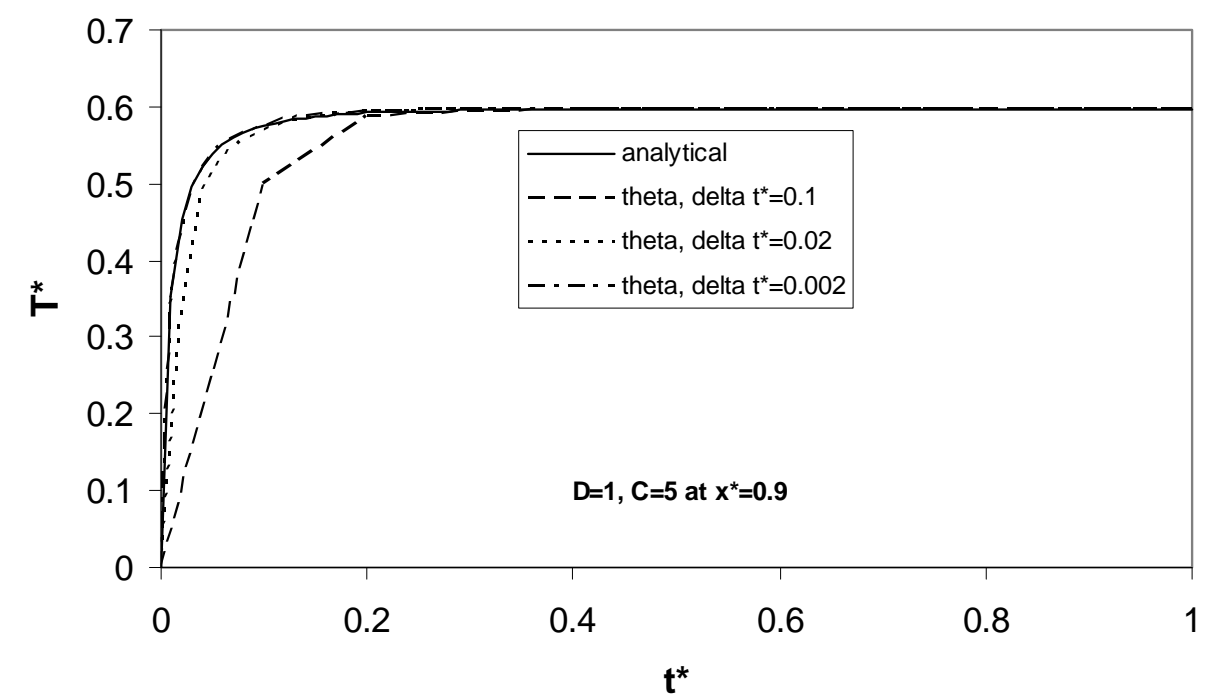

Fig. 15. Transient response of convection-diffusion equation using theta method at $D=1, C=5$ and ${ }^{*}=0.9$.

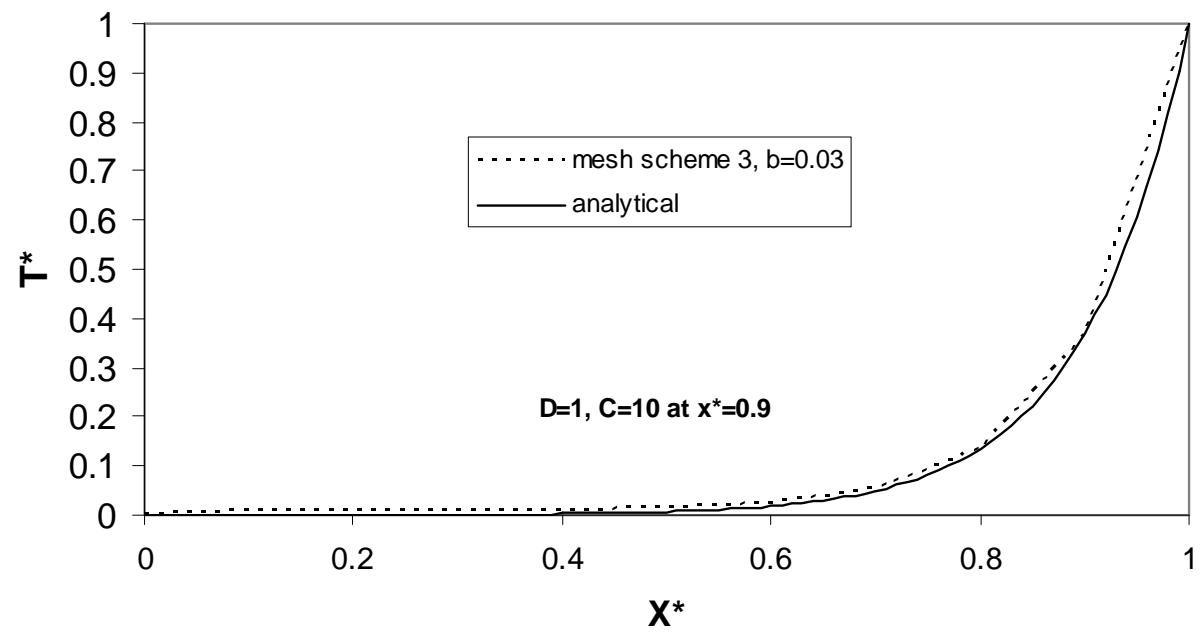

Fig. 16. Steady solution of the convection-diffusion equation at $D=1, C=10$ using mesh scheme 3 with spatial bubble. 


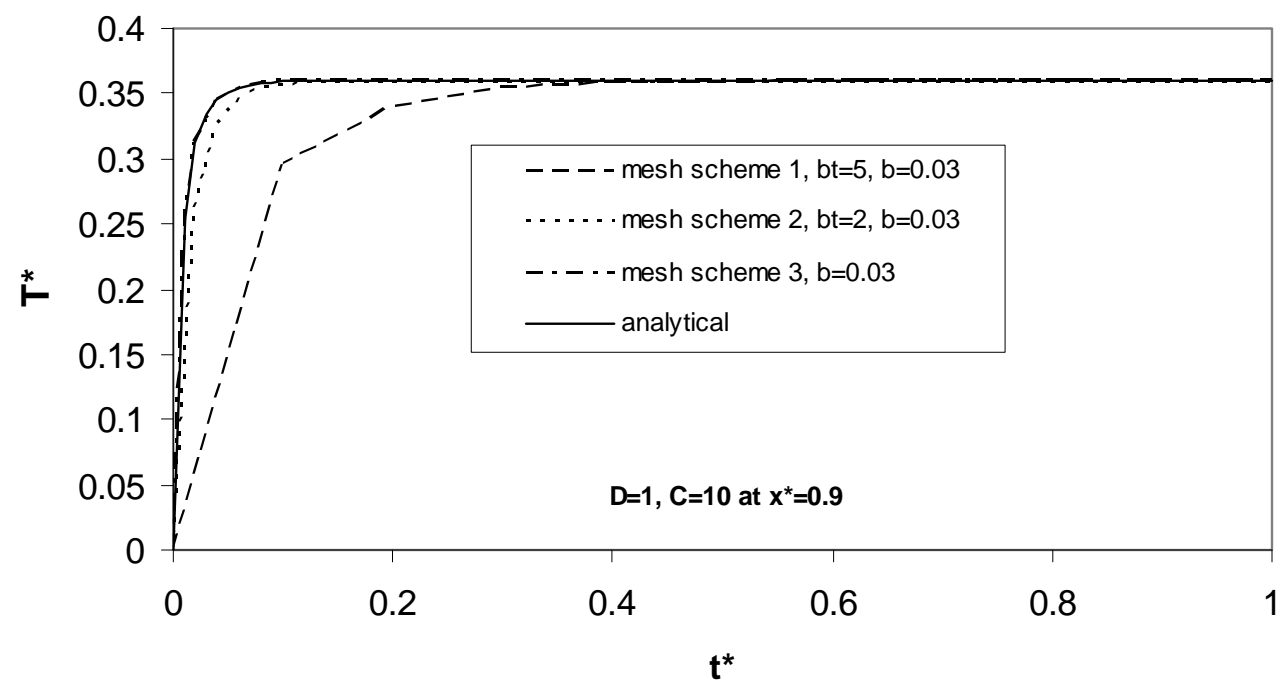

Fig. 17. Transient response of convection-diffusion equation at $D=1, C=10$ and $\mathrm{x}^{*}=0.9$. Mesh schemes 1 and 2 with temporal and spatial bubbles and mesh scheme 3 with just spatial bubble function..

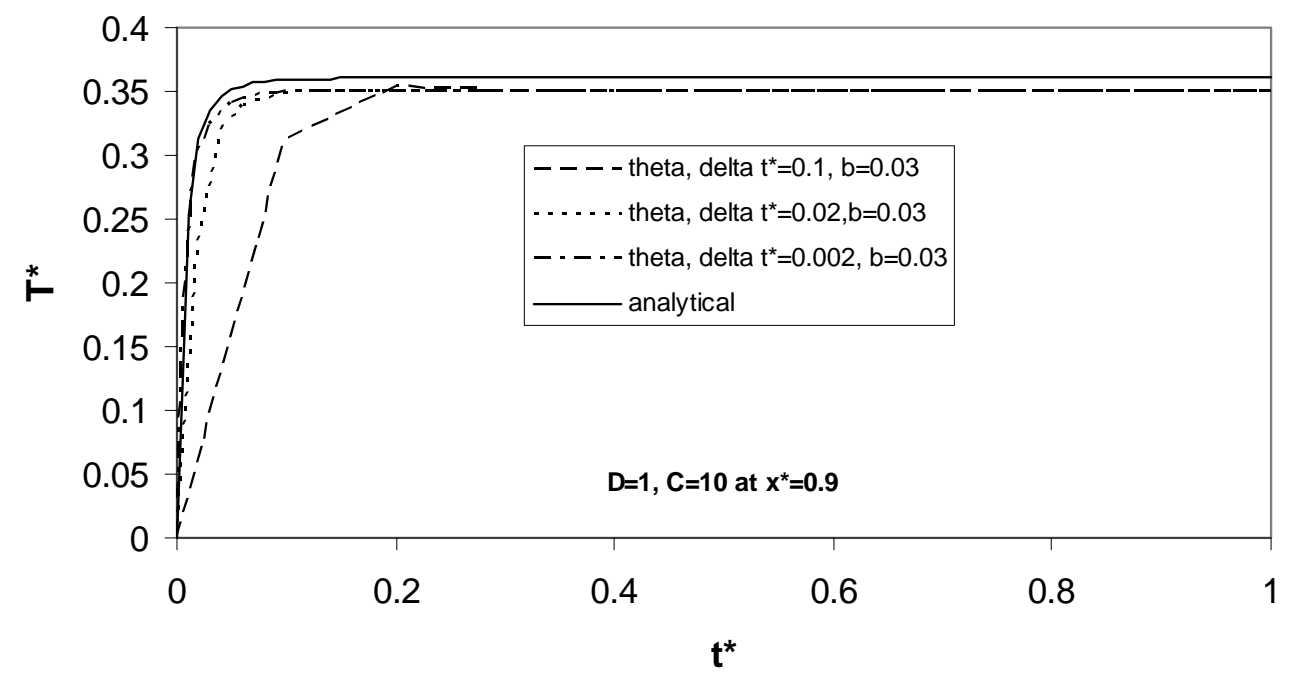

Fig. 18. Transient response of convection-diffusion equation using theta method at $D=1, C=10$ and $\mathrm{x}^{*}=0.9$ with spatial bubble function.. 


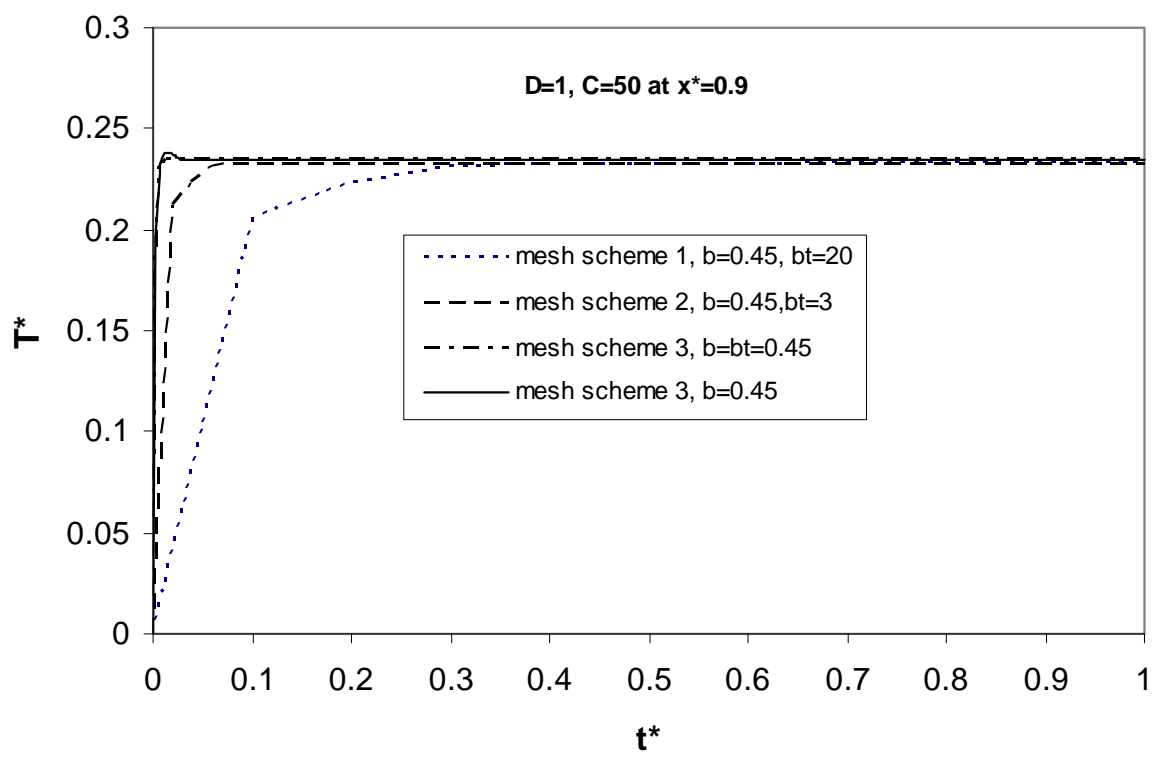

Fig. 19. Transient response of convection-diffusion equation at $D=1, C=50$ and $\mathrm{x}^{*}=0.9$ for all mesh schemes with spatial and temporal meshes..

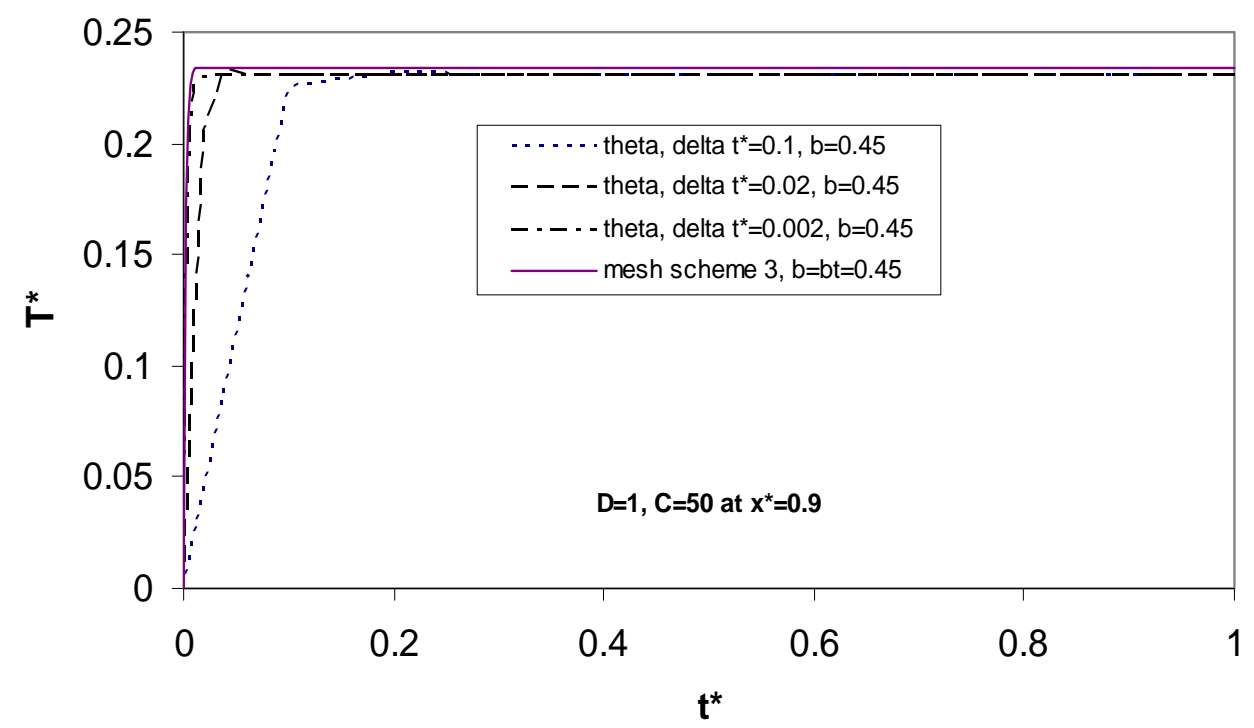

Fig. 20. Comparison of transient response of convection-diffusion equation using theta method at $D=1$, $C=50$ and $\mathrm{x}^{*}=0.9$ with the multiscale method using mesh scheme 3 with both spatial and temporal bubbles. 


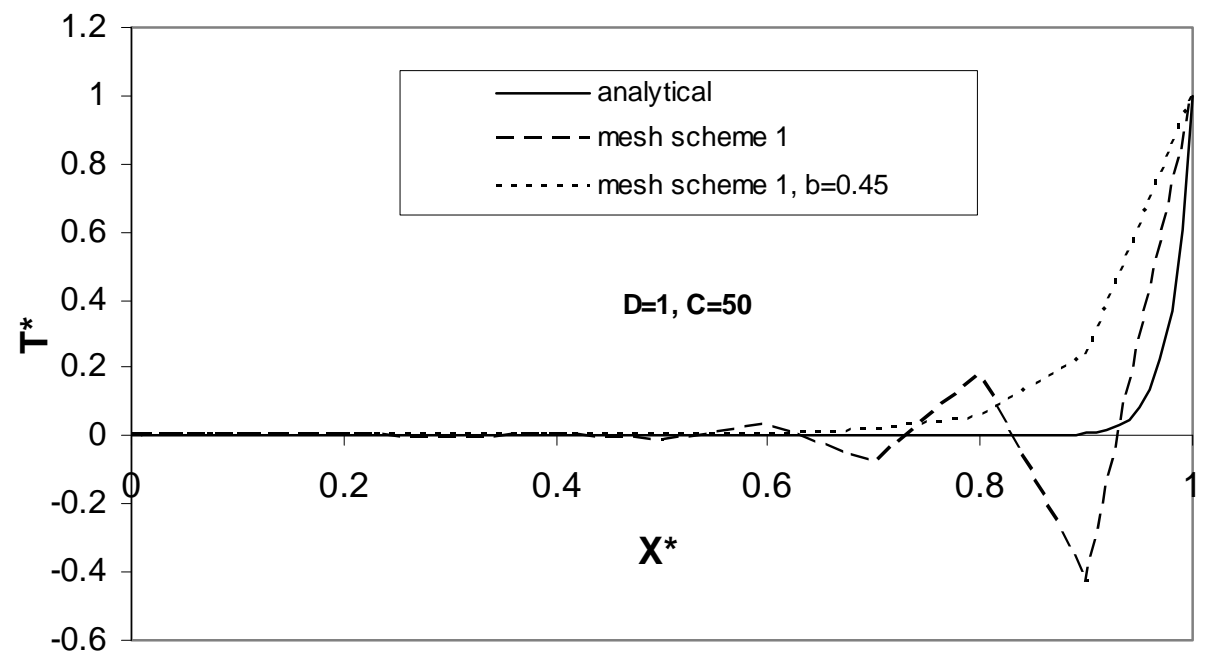

Fig. 21. Steady solution of the convection-diffusion equation at $D=1, C=50$ using mesh scheme 1 with and without spatial bubble.

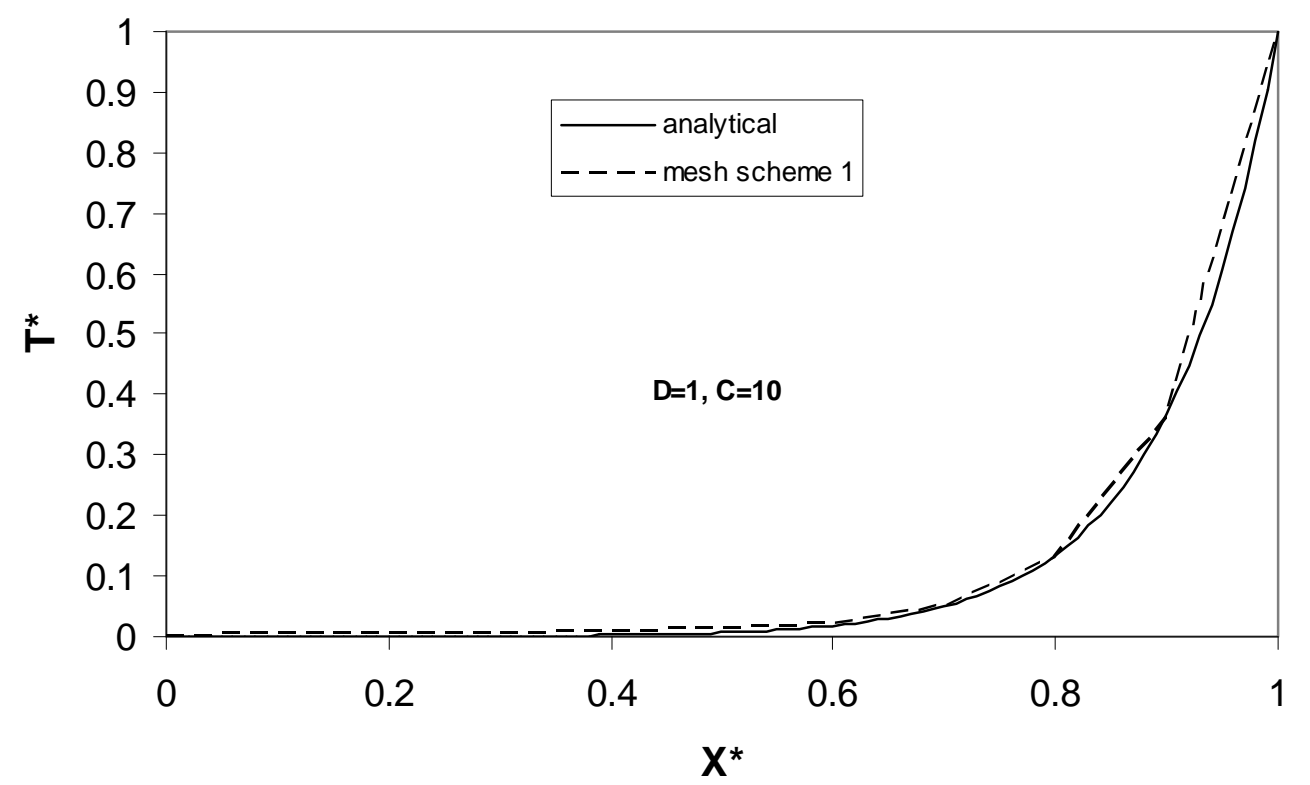

Fig. 22. Steady solution of the convection-diffusion equation at $D=1, C=10$. No multiscale behaviour in $\mathrm{x}$ direction is observed. 


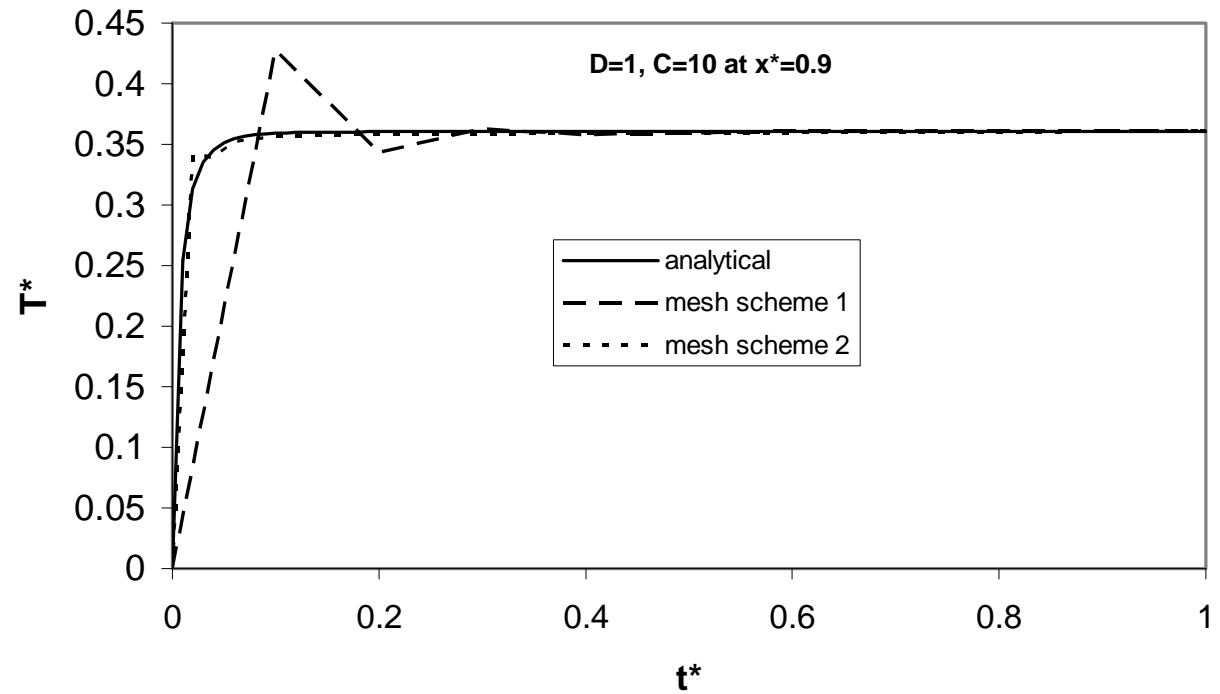

Fig. 23. Transient response of convection-diffusion equation at $D=1, C=10$ and ${ }^{*}=0.9$ with temporal multiscale behaviour.

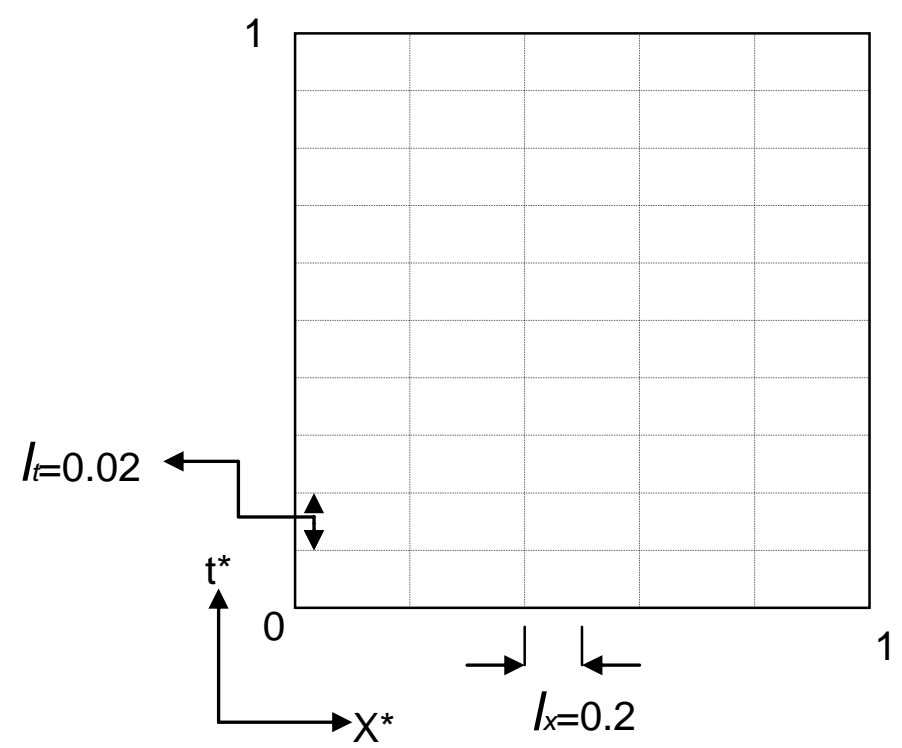

Fig. 24. Mesh scheme 4. 


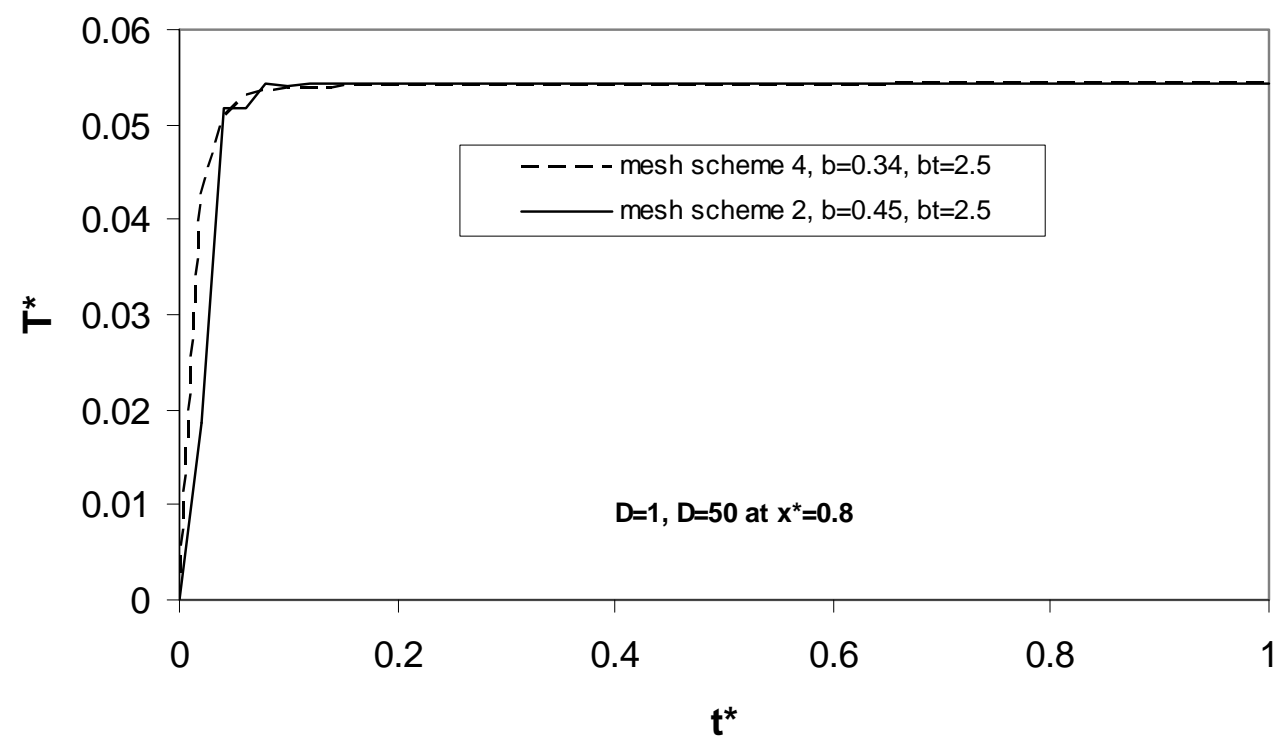

Fig. 25. Transient response of convection-diffusion equation at $D=1, C=50$ and $\mathrm{x}^{*}=0.8$ using different $\mathrm{x}$ refinement of mesh schemes 2 and 4 with both spatial and temporal bubble functions.. 